\title{
Paradoxical fMRI overconnectivity upon chemogenetic silencing of the prefrontal cortex
}

\section{Federico Rocchi}

Italian Institute of Technology

\section{Carola Canella}

Italian Institute of Technology

Shahryar Noei

Italian Institute of Technology

Daniel Gutierrez-Barragan

Italian Institute of Technology

\section{Ludovico Coletta}

Italian Institute of Technology

\section{Alberto Galbusera}

Istituto Italiano di Tecnologia https://orcid.org/0000-0001-7213-0013

\section{Stefano Vassanelli}

University of Padova https://orcid.org/0000-0003-0389-8023

\section{Massimo Pasqualetti}

University of Pisa https://orcid.org/0000-0002-0844-8139

Giuliano lurilli

Italian Institute of Technology

\section{Stefano Panzeri}

Istituto Italiano di Tecnologia https://orcid.org/0000-0003-1700-8909

Alessandro Gozzi ( $\nabla$ alessandro.gozzi@iit.it )

Italian Institute of Technology

\section{Article}

Keywords: fMRI, Paradoxical fMRI overconnectivity

Posted Date: January 18th, 2021

DOI: https://doi.org/10.21203/rs.3.rs-140535/v1

License: (c) (i) This work is licensed under a Creative Commons Attribution 4.0 International License. Read Full License 
Version of Record: A version of this preprint was published at Nature Communications on February 25th, 2022. See the published version at https://doi.org/10.1038/s41467-022-28591-3. 
Paradoxical fMRI overconnectivity upon chemogenetic silencing of the prefrontal cortex

Federico Rocchi ${ }^{1,2^{*}}$, Carola Canella ${ }^{1,2^{*}}$, Shahryar Noei ${ }^{2,3^{*}}$, Daniel Gutierrez-Barragan ${ }^{1}$, Ludovico Coletta ${ }^{1,2}$, Alberto Galbusera ${ }^{1}$, Stefano Vassanelli ${ }^{4}$, Massimo Pasqualetti ${ }^{1,5}$, Giuliano lurilli ${ }^{6}$, Stefano Panzeriं ${ }^{3 \S}$, Alessandro Gozzi ${ }^{1 \S}$

*Equal first-author contribution

$\S$ Senior authors

${ }^{1}$ Functional Neuroimaging Laboratory, Center for Neuroscience and Cognitive systems, Istituto Italiano di Tecnologia, Rovereto, Italy

${ }^{2}$ Center for Mind and Brain Sciences, University of Trento, Rovereto, Italy

${ }^{3}$ Neural Computational Laboratory, Center for Neuroscience and Cognitive Systems, Istituto Italiano di Tecnologia, Rovereto, Italy

${ }^{4}$ University of Padua, Italy

${ }^{5}$ Biology Department, University of Pisa, Pisa, Italy

${ }^{6}$ Systems Neurobiology Laboratory, Center for Neuroscience and Cognitive systems, Istituto Italiano di Tecnologia, Rovereto, Italy

\section{Correspondence}

Alessandro Gozzi, PhD

Functional Neuroimaging Laboratory

Istituto Italiano di Tecnologia

Center for Neuroscience and Cognitive Systems @ UNITN

38068, Rovereto, Italy

alessandro.gozzi@iit.it

Running title: Cortical silencing leads to fMRI overconnectivity 


\section{ABSTRACT}

While shaped and constrained by axonal connections, fMRI-based functional connectivity can reorganize in response to varying interareal input or pathological perturbations. However, the causal contribution of regional brain activity to whole-brain $\mathrm{FMRI}$ network organization remains unclear. Here we combine neural silencing, resting-state fMRI and in vivo electrophysiology to causally probe how inactivation of a cortical node affects brain-wide fMRI coupling in the mouse. We find that chronic suppression of the medial prefrontal cortex (PFC) via overexpression of a potassium channel paradoxically increases $\mathrm{fMRI}$ connectivity between the silenced area and its direct thalamo-cortical terminals. Acute chemogenetic inactivation of the PFC reproduces analogous patterns of fMRI overconnectivity, with increased fMRI coupling between polymodal thalamic regions and widespread cortical areas. Using multielectrode electrophysiological recordings, we further show that chemogenetic inactivation of the PFC results in enhanced slow $(0.1-4 \mathrm{~Hz})$ oscillatory coupling between $\mathrm{FMRI}$ overconnected areas, and that changes in $\delta$ band coherence are linearly correlated with corresponding increases in $\mathrm{fMRI}$ connectivity. These results provide causal evidence that cortical inactivation does not necessarily lead to reduced inter-areal coupling, but can counterintuitively increase fMRI connectivity via enhanced, less-localized slow oscillatory processes, with important implications for modelling and understanding fMRI overconnectivity in pathological states. 


\section{Introduction}

A rapidly expanding approach to understand neural organization is to map large-scale patterns of neural activity in the resting brain via non-invasive neuroimaging. The ease and reproducibility of resting state $\mathrm{fMRI}$ (rsfMRI) have promoted the widespread use of this approach, leading to the observation that spatio-temporal patterns of spontaneous fMRI activity are organized into highly coherent functional networks, defined by temporally correlated fluctuations in BOLD signal ${ }^{1}$. The non-invasive nature of rsfMRI has fueled the use of this method to map intrinsic brain network organization in the healthy human brain, as well as in psychiatric or neurological conditions, in which evidence of disrupted or aberrant rsfMRI functional coupling has been largely documented ${ }^{1}$. However, despite the growing popularity of rsfMRI, our knowledge of the underpinnings of brainwide $\mathrm{fMRI}$ coupling remains very limited.

Multiple lines of evidence suggests that structural and rsfMRI-based connectivity are robustly related ${ }^{1}$. First, structural and functional connection strengths are correlated both at the whole-brain and mesoscopic scale ${ }^{2-4}$, and rsfMRI network topography closely recapitulates patterns of anatomical connectivity in several mammalian species ${ }^{4-6}$. Second, experimental resection of callosal connections ${ }^{7}$ or chemogenetic inactivation of the amygdala result in reduced rsfMRI connectivity with regions anatomically linked to the manipulated $\operatorname{areas}^{8}$. Lastly, computational modelling corroborates a tight relationship between structural and functional connectivity, as synchronous rsfMRI fluctuations can be modelled by dynamical systems endowed with realistic anatomical connectivity patterns of longrange axonal interactions ${ }^{9}$. Accordingly, simulated axonal lesions in these models result in reduced functional coupling ${ }^{10}$.

These observations have prompted the widespread use of statistical dependencies in spontaneous fMRI signal as an index of interareal functional communication ${ }^{1}$. However, the neural basis and foundational principles linking regional brain activity to brain-wide rsfMRI network connectivity remain unclear. For example, growing experimental evidence suggest that, while tightly constrained by underlying anatomy, rsfMRI connectivity may only partially reflect direct interactions between areas, as widespread BOLD signal modulation might arise via subcortical connections whether through the thalamus via long-range loops ${ }^{11}$, or as a result of diffuse neuromodulation mediated by brainstem nuclei ${ }^{12-14}$. This notion is epitomized by the observation of intact rsfMRI coupling among brain regions not directly structurally connected as in the case of acallosal humans, primates and rodents $7,15,16$. Moreover, rsfMRI network topography can dynamically reconfigure in response to 
local perturbations ${ }^{17}$ or pathological processes ${ }^{18}$. In keeping with this, neurological disorders such as Parkinson's disease, stroke and Alzheimer's disease have been often found to be associated with unexpectedly increased interareal rsfMRI connectivity despite the loss of cortical function characterizing these conditions 19,20 . Taken together, these observations point at a complex relationship between interareal brain activity and rsfMRI coupling, and call for a deeper investigation of the principles governing the establishment of rsfMRI network connectivity and its reconfiguration in response to varying interareal input or pathological perturbations.

Here we combine rsfMRI, neural and chemogenetic silencing (chemo-fMRI ${ }^{21}$ ) and in vivo electrophysiology in the mouse to probe how inactivation of a cortical area causally affects rsfMRI coupling. Surprisingly, we find that chronic and acute neural silencing of the medial prefrontal cortex (PFC), a core component of the mouse default mode network DMN, 22 may lead to increased rsfMRI coupling between the silenced area and its thalamo-cortical terminals. This effect is associated with decreased $\curlyvee$ power in the suppressed region and robustly increased low-frequency $(0.1-1 \mathrm{~Hz})$ and $\delta$ band (1-4 Hz) electrophysiological coherence between functionally overconnected PFC terminals. These findings reveal a highly dynamic, non-monotonic relationship between regional cortical activity and network-wide rsfMRI connectivity and provide an interpretative framework for the observation of counterintuitively increased rsfMRI connectivity in pathological conditions characterized by impaired cortical function. 


\section{Results}

\section{rsfMRI overconnectivity upon chronic inactivation of the mouse prefrontal cortex}

The robust structural foundations of rsfMRI connectivity suggest that neural silencing of a network node would result in diminished functional coupling with regions receiving direct axonal projections from the inactivated region ${ }^{8,10,23}$. To test this prediction and more broadly investigate how rsfMRI dynamically reconfigures in response to local neural suppression, we carried out rsfMRI measurements in a cohort of mice in which neuronal activity in the medial prefrontal cortex (abbreviated here as PFC) was chronically inhibited via a bilateral viral transduction of the inward rectifying potassium channel Kir2.1 (Fig. S1A). Prior research has shown that virally-mediated Kir2.1 expression results in a reduction of both evoked and spontaneous neuronal excitability lasting several weeks ${ }^{24,25}$. In keeping with this, in vivo electrophysiological recordings in the PFC of mice unilaterally transfected with Kir2.1 revealed a robust reduction of spontaneous firing rate in the targeted cortical area with respect to its control contralateral regions ( $N=4$, paired t test, $p=0.002$, Fig. S2).

We next compared the patterns of rsfMRI connectivity in Kir2.1 and GFP-transduced control littermates, by imaging Kir2.1 transfected mice four weeks after viral injections (Fig. 1A). Consistent with previous investigations 26,27 , seed-based probing revealed significant long-range correlation between the PFC and thalamo-cortical components of the mouse DMN in both cohorts (Fig. 1B). Surprisingly, between-group comparisons revealed foci of significantly increased rsfMRI connectivity in the posterior cingulate/retrosplenial cortex and centromedial thalamic regions of Kir2.1 transfected mice (t test, $p<0.05, t>2.03$, FWE cluster-corrected, $p<0.05$; Fig. 1C). Regional quantifications of DMN connectivity via multiple prefrontal-DMN seeds corroborated these findings, revealing increased rsfMRI synchronization along the entire midline extension of this network (twoway ANOVA, $F(1,33)=6.93 ; p=0.013 ;$ Fig. $1 D)$ and its centromedial thalamic targets (t test, $t 33=$ 2.589, $p=0.014$; Fig. 1E). Voxel-wise mapping did not reveal any foci of reduced functional connectivity with the PFC ( $\mathrm{t}>2.03$, cluster correction at $\mathrm{p}<0.05)$. Importantly, all the thalamic and cortical regions showing increased rsfMRI connectivity in Kir2.1 mice are characterized by high axonal projection density from the PFC, as seen by comparing the magnitude of inter-group rsfMRI connectivity differences with incoming axonal connectivity strength inferred from a voxel-model of the mouse brain connectome ${ }^{4}$ (Fig. 1F, Wilcoxon rank sum test, $\mathrm{p}<0.0001$ ). Interestingly, the direction and anatomical location of DMN rsfMRI overconnectivity was not altered by global fMRI signal regression (Fig. S3), with the exception of thalamic areas, in which the connectivity difference 
between groups was attenuated. Together, these findings reveal that chronic inhibition of neural activity in the PFC may counterintuitively increase rsfMRI functional connectivity between long-range thalamo-cortical terminals of the mouse DMN.

A

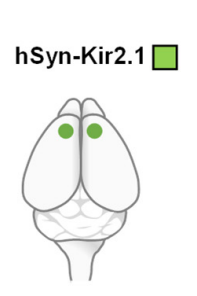

C

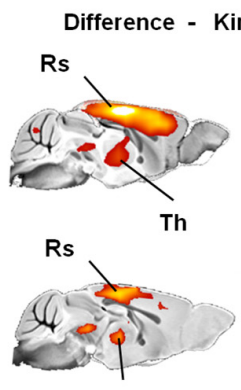

Th
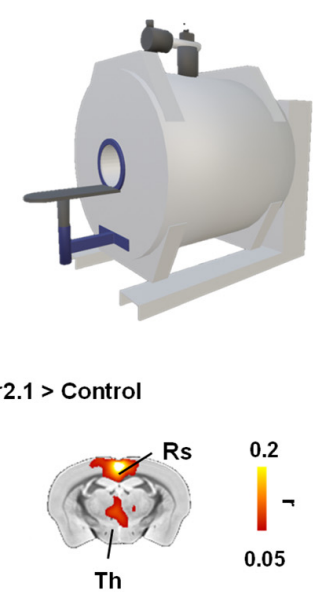

Th

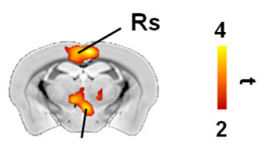

Th
B

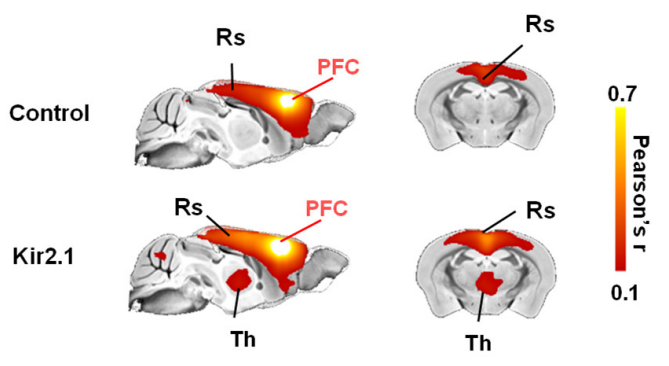

D

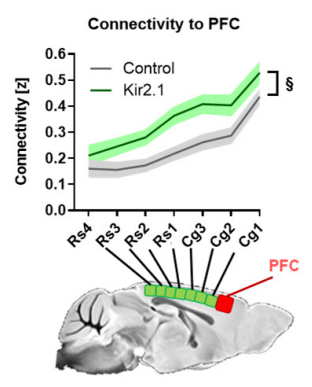

$\mathrm{E}$

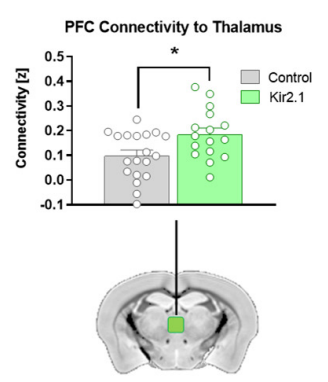

$\mathbf{F}$
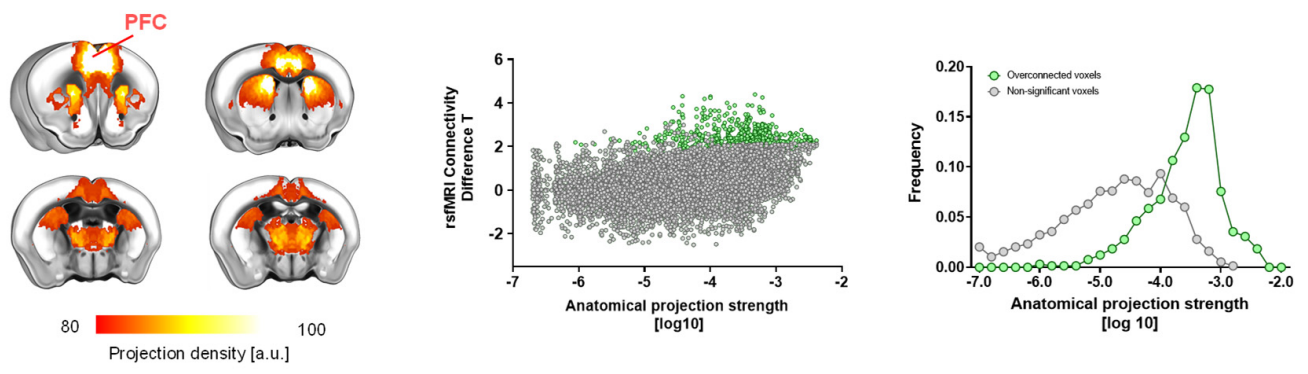

Figure 1

Chronic silencing of the mouse PFC results in rsfMRI overconnectivity (A) Summary of experimental design. The potassium channel Kir2.1 $(n=16)$ or GFP (control, $n=19)$ were transduced bilaterally into the PFC of adult male mice. Four weeks after viral injections, mice underwent rsfMRI scanning. (B) Seed based connectivity mapping of the PFC in GFP (control), and Kir2.1-transduced subjects. (C) Corresponding group difference maps. Area with significantly increased rsfMRI connectivity in Kir2.1 expressing mice are depicted in red-yellow ( $r$ and T stat difference map). (D) Antero-posterior profiling of rsfMRI connectivity of the PFC within the midline axis of the mouse DMN. (E) Fronto-thalamic rsfMRI overconnectivity in Kir2.1 expressing mice. (F) Regions exhibiting rsfMRI overconnectivity in Kir2.1 mice are robustly innervated by the PFC. Left: Axonal projections from the PFC (top 20\% strongest connections). Middle: scatter plot illustrating intergroup differences in rsfMRI connectivity as a function of PFC structural connectivity strength. Green dots indicate significantly functionally overconnected voxels. Right: Distribution of overconnected voxels as a function of axonal connectivity strength Green. DMN: Default Mode Network; Cg: cingulate cortex; PFC: prefrontal cortex, RS: 
retrosplenial; Th: Thalamus. $\S p<0.05,2$-way ANOVA, repeated measurements; * $p<0.05$, student $t$ test.

\section{rsfMRI overconnectivity upon acute chemogenetic inactivation of the mouse prefrontal cortex}

To corroborate the specificity of Kir2.1 findings and obtain mechanistic insight into the neural correlates of the observed fMRI overconnectivity, we designed a new set of experiments in which DREADD-based chemogenetics was employed to induce a time-controlled, acute silencing of PCF activity during rsfMRI scanning. An overview of experimental procedures is provided in Figure $2 \mathrm{~A}$. To enable remote silencing of fronto-cortical activity, we bilaterally transfected the PFC with the inhibitory hM4Di DREADD using a pan-neuronal promoter (Figure 2A), a strategy widely used to regionally suppress neural function in behavioral studies ${ }^{28}$. Three weeks after viral injection, control (GFP-transfected) and hM4Di-expressing animals underwent rsfMRI scanning or electrophysiological recordings before and after intravenous injection of the DREADD activator clozapine-N-oxide (CNO). To account for the relatively slow pharmacokinetic profile of $\mathrm{CNO}$ in the rodent brain ${ }^{29}$, 30, both imaging and electrophysiological recordings were split into a pre-CNO injection baseline, a transitory (0 -15 min) drug-equilibration period, and an active time window (15-50 min post CNO injection) to which all our analyses refer to, unless otherwise specified (Fig. 2B-C).

To assess whether acute chemogenetic silencing of the PFC results in rsfMRI oversynchronization, we compared rsfMRI connectivity patterns in hM4Di transfected and control mice upon acute CNO administration (active phase, Fig. 2 and Fig. S1B). Recapitulating the Kir2.1 findings, voxel-wise mapping revealed foci of significantly increased rsfMRI connectivity in the posterior cingulate/retrosplenial cortices and midline thalamic regions of DREADD-expressing mice (t test, $\mathrm{P}<0.05$, FWE corrected, cluster defining threshold $\mathrm{t}>2.03, \mathrm{p}<0.05$; Fig. 2D). Regional quantifications corroborated the presence of rsfMRI overconnectivity along the cingulate and retrosplenial axis of the DMN, and between the PFC and medio-dorsal thalamic areas (2-way repeated measures ANOVA, $F_{1,32}=6.58 ; p=0.0155 ; t_{\text {test }} t_{32}=4.30, p=0.001$, respectively; Fig. 2EF), a set of regions characterized by dense incoming projections from the PFC (Fig. S4, Wilcoxon rank sum test, $\mathrm{p}<0.0001$ ). Notably, the direction and the anatomical location of the observed rsfMRI overconnectivity was regionally unaltered by global fMRI signal regression (Figure S3), arguing against an unspecific contribution of arousal related global dynamics or global fMRI co-activation to the mapped changes ${ }^{13,14,31}$. 
A

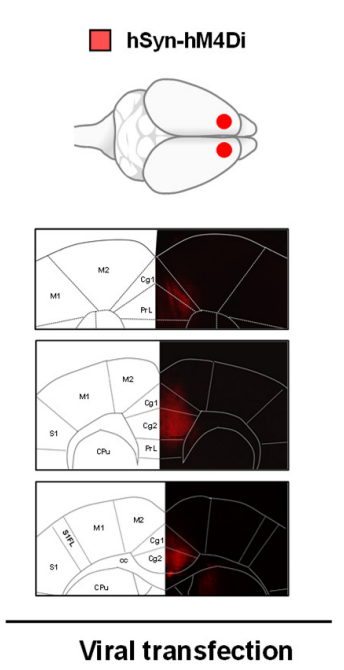

B

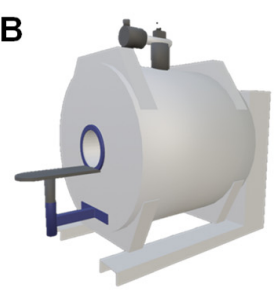

C
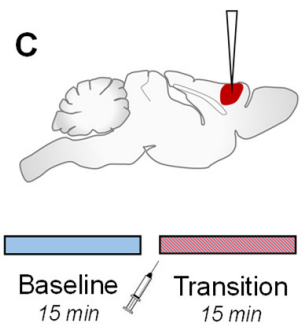
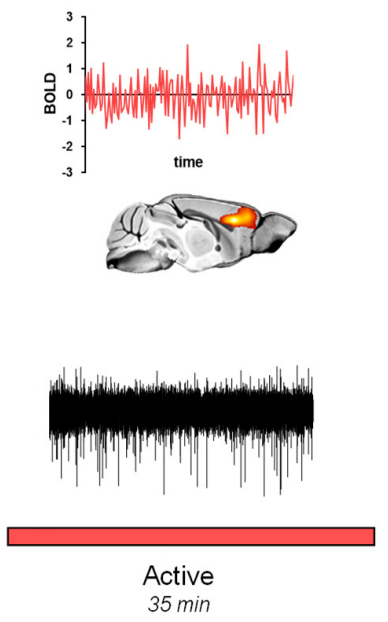

rsfMRI and electrophysiological recordings
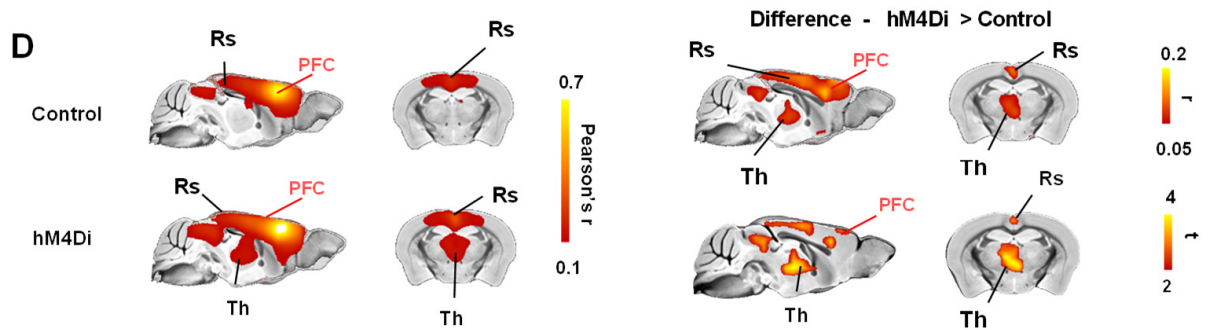

E

$\mathbf{F}$
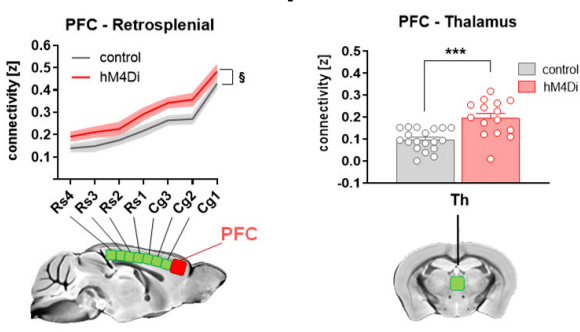

G
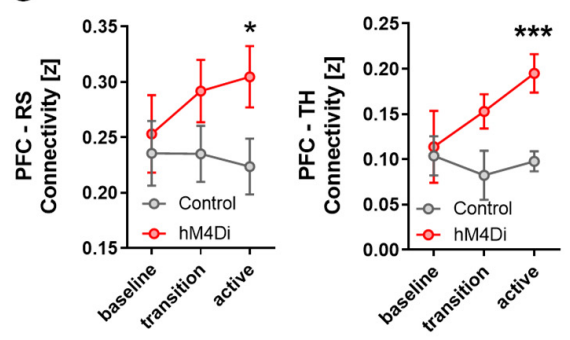

Figure 2

Chemogenetic silencing of the mouse PFC results in rsfMRI overconnectivity. (A) Experimental design of chemo-fMRI experiments. AAV8-hSyn-hM4Di $(n=15)$ or AAV8-hSyn-GFP (control, $n=19)$ were bilaterally injected into the PFC of wild type. The bottom panels show representative viral expression across three adjacent PFC slices three weeks after transduction (B) mice underwent rSfMRI scanning or (C) electrophysiological recordings. A reference acquisition timeline is reported to depict timeseries binning into a 15 min pre-CNO reference baseline, a drug equilibration window (15 min, transition), and a 35 min CNO active time window (active). (D) Seed-based connectivity of the PFC and between group difference map revealed rsfMRI over-connectivity in the DMN of hM4Di expressing mice during the active phase. (E) Antero-posterior profiling of rsfMRI connectivity of the PFC along the midline axis of the mouse DMN in the two cohorts. (F) Thalamo-cortical rsfMRI hyper synchronization in hM4Di expressing mice. (G) Prefrontal-retrosplenial and prefrontal-thalamic connectivity timecourse. Data are plotted as mean \pm SEM. Cg: cingulate cortex; PFC: prefrontal cortex, RS: retrosplenial cortex; Th: Thalamus. \& $p<0.05,2$-way ANOVA repeated measurements, genotype effect; ${ }^{*} p<0.05, * * *<p<0.001$, student $t$ test. 
Baseline PFC connectivity in these areas was comparable across groups (voxel-wise mapping, $Z>2.03$ cluster corrected, PFC-Cingulate, 2-way ANOVA, $F_{1,32}=0.48, p=0.49$, Thalamo-PFC, $t$ test, $t_{32}$ $=0.23, p=0.81$ ), and oversynchronization gradually emerged in the hM4Di cohort after CNO administration, peaking during the DREADD active time-window (PFC-Rs: $T_{32}=2.158, p=0.03$, PFCTh: $T_{32}=4.301, p=0.0001$, student $t$ test, Fig. 2G). Moreover, no intergroup differences were observed in the estimated characteristic hemodynamic response function in this area (kernel height $p>0.6$; time-to-peak $p>0.12$, full-width-at-half-peak $p>0.37$, t test) nor were between-group differences in arterial blood pressure $\left(p>0.7\right.$, Student t test) or blood gas levels observed $\left(\mathrm{P}_{a} \mathrm{CO}_{2} p=\right.$ 0.49; $\mathrm{P}_{\mathrm{a}} \mathrm{O}_{2} \mathrm{p}=0.22$, Student t-test). These control measurements rule out major spurious vascular or hemodynamic contributions and corroborate the specificity of the mapped changes. Taken together, our chemo-fMRI results show that acute inhibition of PFC activity results in a pattern of DMN oversynchronization closely recapitulating that observed with chronic Kir2.1-mediated silencing, suggesting that the ensuing overconnectivity is not manipulation-specific, nor the indirect consequence of homeostatic reactivity to protracted neural silencing.

\section{Chemogenetic silencing of the prefrontal cortex leads to thalamo-cortical rsfMRI oversynchronization}

Topographical mapping of rsfMRI connectivity upon chemogenetic silencing of the PFC revealed foci of hyperconnectivity in medio-dorsal and centro-medial areas of the thalamus, a set of higher order nuclei densely innervated by the mouse PFC ${ }^{32}$. We thus probed the rsfMRI connectivity of the thalamus to assess whether the observed foci of thalamic overconnectivity could underlie or involve the engagement of additional brain regions outside the DMN. To this aim, we first clustered thalamic voxels based on their unique between-group functional connectivity profile differences as previously described ${ }^{33}$. Consistent with the neuroanatomical organization of this region, our approach revealed two segregable thalamic sub-territories, one encompassing its centromedial and anterodorsal (PFC-innervated) polymodal components, and the second encompassing more lateral (unimodal/sensory) areas (Fig. 3A). This anatomical segregation is of potential mechanistic interest, as polymodal thalamic areas have been recently shown to serve as key generators and cortical propagators of slow neural rhythms (e.g. $\delta$ ) relevant to rsfMRI coupling ${ }^{11,34-36}$. 

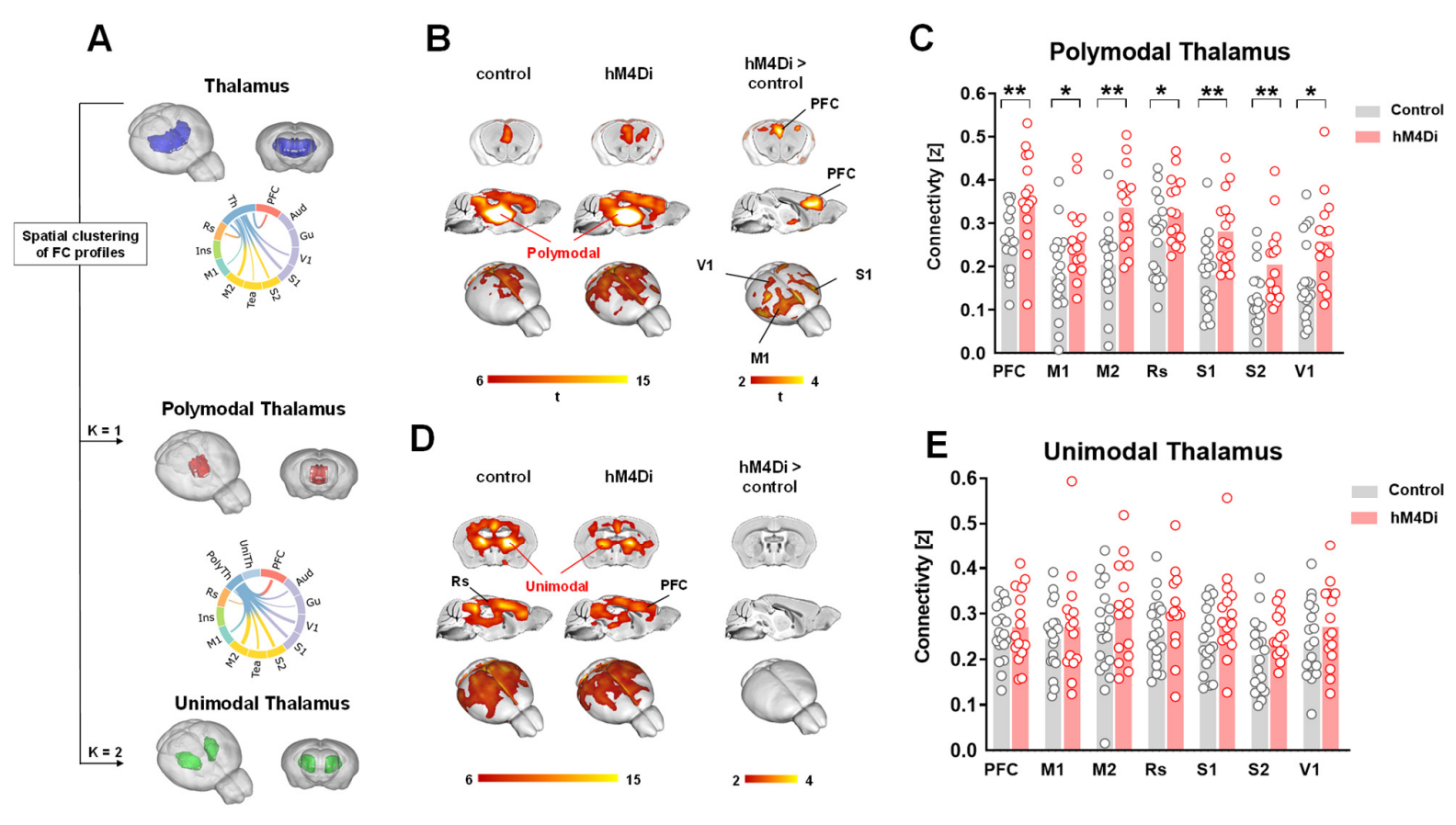

Figure 3

The polymodal thalamus is functionally overconnected to cortical areas. (A) k-means clustering of PFC-thalamic rsfMRI connectivity profiles (thalamus, blue; unimodal thalamus, green; polymodal thalamus, red) and circular representation of between-group connectivity difference of the entire thalamus and thalamic partition (UniTh: unimodal; PolyTh: polymodal thalamus) in Control ( $n=19$ ) and $h M 4 D i(n=15)$ animals. (B-D) Seed connectivity of sub-thalamic partitions, between group difference maps (B-C polymodal thalamus; $D-E$, unimodal thalamus) and corresponding quantification of thalamo-cortical connectivity ( ${ }^{*} p<0.05,{ }^{* *} p<0.01$; Benjamini-Hochberg corrected).

Interestingly, seed-based probing of the unimodal thalamus did not reveal significant rsfMRI connectivity differences between hM4Di-expressing and control animals (Fig. 3D-E; Z> 2.03, all voxels, cluster corrected at $t>2.03)$, whereas seed-based probing of the polymodal thalamus revealed in hM4Di expressing-mice a widespread pattern of cortical overconnectivity, exceeding the boundaries of the PFC to encompass motor and somatosensory territories, including the retrosplenial cortex. To probe the network specificity of these effects and rule out the presence of generalized overconnectivity in hM4Di mice, we next mapped whole-brain rsfMRI connectivity in hM4Di and control animals using a whole-brain parcellation scheme (Fig. S5). This analysis revealed the presence of significant clusters of rsfMRI overconnectivity only in regions of the DMN and in polymodal thalamic areas of DREADD-expressing animals, the latter areas exhibiting oversynchronization with larger cortical regions. No meaningful clusters of rsfMRI over- or under-connectivity were otherwise observed in any of the area probed. These results show that, upon acute suppression of fronto- 
cortical activity, PFC-innervated polymodal thalamic regions, but not unimodal areas, exhibit oversynchronous rsfMRI coupling with large cortical areas.

\section{Chemogenetic silencing of the prefrontal cortex increases interareal slow oscillatory coherence}

To test the efficacy of chemogenetic silencing and investigate the neural correlates of the observed rsfMRI overconnectivity, we performed electrophysiological recordings in the PFC of hM4Di- or GFP-transduced control animals prior to and after CNO administration, under the same experimental conditions of rsfMRI (Fig. 4). Baseline electrophysiological traces revealed the presence of appreciable spontaneous multi-unit activity in the PFC both groups (mean firing rate $15.0 \pm 2.2$ spikes/s in hM4Di-expressing, and $14.1 \pm 3.8$ in GFP-transduced mice, $n=5$ each group, $p=0.85, t$ test, Fig. $4 A B$ ). As expected, CNO administration robustly inhibited firing rate in hM4Di expressing mice, but not in control subjects (Fig. 4A-C, p<0.01 FDR corrected, $t$ test). In keeping with the timeprofile of rsfMRI overconnectivity, DREADD-induced PFC inhibition was characterized by gradual decrease of neural firing upon CNO administration, reaching a steady state approximately 10-15 min after the intravenous bolus (Fig. 4C).

To obtain insight into the neural rhythms underlying the observed rsfMRI overconnectivity, we next analyzed local field potential (LFP) traces obtained from the same set of PFC electrophysiological recordings. Consistent with the observed firing rate reduction, LFP spectrograms from hM4Di-transduced animals revealed a robust decrease in $\beta$ - and $y$-band power after CNO administration with respect to $C N O$ treated controls $\left(F_{1,22}=239.4, p<0.1\right.$ and $p<0.001$, respectively, Fig. 4E). However, the LFP power reduction in these frequency bands was also accompanied by a prominent increase in $\delta$ - and slow-band (defined here as 0.1 - $1 \mathrm{~Hz}$ ) LFP power in DREADD-expressing subjects (Fig. 4C-D, slow $p<0.001, \delta p<0.01, \theta p=0.77 ; \alpha p=0.34 ; \beta p=0.01 ; y p<0.001 ;$ Student $t$ test, Bonferroni corrected).

The observed increase in slow and $\delta$ band LFP power in the chemogenetically silenced PFC suggest that that the resulting rsfMRI overconnectivity could be driven by enhanced low-frequency neural coherence as opposed to direct interareal communication via higher frequency neural oscillations ${ }^{37-39}$. To test this hypothesis, we carried out a set of simultaneous multi-electrode LFP recordings in the PFC, retrosplenial and centromedial (polymodal) thalamus of control and DREADDtransfected animals (Fig. 3). We selected these region pairs because they exhibit the highest functional overconnectivity in our chemo-fMRI study. Spectral-power quantifications after CNO administration revealed a reduction of $\gamma$ and $\beta$ LFP power in all the three recording sites, together 
with increased slow and $\delta$ LFP power in the PFC, but not in the retrosplenial cortex or centromedial thalamus of DREADD-transfected animals (Fig. S6A). To explore the possible neural correlates of fMRI connectivity in terms of frequency-dependent neural communication, we next computed LFP power coherence between electrophysiological signals recorded at these electrode pairs. Notably, we found a clear CNO-induced increase in low-frequency LFP power coherence in PFC-Rs and PFC-polymodal thalamic areas in hM4Di-transfected animals (Fig. S6B). In keeping with rsfMRI evidence of increased thalamo-cortical connectivity upon PFC silencing (Fig. 3C), enhanced low-frequency power coherence at was also observed between the thalamus and retrosplenial cortex (Fig. S6B). A quantification of these changes in canonical LFP frequency bands revealed significantly increased power coherence in the slow and $\delta$ bands for all the three electrode pairs (Fig. 4F-H, q $<0.05$, FDR corrected, all pairs). We next computed inter-areal coherence between the envelope of $\curlyvee$-band amplitude, because ultraslow $(\sim 0.1 \mathrm{~Hz})$ variation in $\curlyvee$-band envelope has been linked to arteriole dynamics and suggested to be a contributing factor to rsfMRI coupling ${ }^{36,40}$. Mean coherence plots of $\curlyvee$-envelope over the ultraslow frequency range $\left(0.02-0.5 \mathrm{~Hz}{ }^{40}\right)$ revealed only marginally increased $\mathrm{CNO}$-induced coherence in hM4Di expressing mice (Fig. S7). In keeping with this observation, we did not find evidence of significantly increased ultra-slow $\curlyvee$-envelope coherence (integrated between 0.02 to $0.5 \mathrm{~Hz}$ ) between any electrode pairs ( $q>0.1$, FDR corrected).

To link these oscillatory changes to the observed rsfMRI patterns, following the procedure described in Wang et al. ${ }^{41}$, we next compared the obtained electrophysiological coherence values with corresponding (separately-measured) interareal rsfMRI connectivity in hM4Di and control animals (Fig. 4I-M). We first compared between-group band-specific LFP coherence differences across all electrode pairs with corresponding interareal rsfMRI connectivity changes measured during the active CNO phase (Fig. 4I). These quantifications revealed that $\delta$ and slow LFP bands were the only frequency ranges exhibiting robustly CNO-induced increased coherence between PFCretrosplenial and PFC-thalamic electrode pairs, recapitulating corresponding CNO-induced interareal rsfMRI increases (Fig. 3I). While considerably smaller, between-group ultra-slow LFP gammaenvelope coherence also revealed CNO-induced increases in all the three electrode pairs (Fig. 3I). Other LFP bands did not show full concordance with rsfMRI findings: for example $\beta$ band coherence increased after CNO administration in PFC-retrosplenial but not in PFC-thalamus electrode pairs. Similarly, no meaningful between-group changes in power coherence upon DREADD stimulation was observed in the $\theta$ and $\gamma$ LFP bands (Fig. 3I). Further supporting a key contribution of slow and $\delta$ 
rhythms to the observed rsfMRI overconnectivity, we found that pairwise LFP coherence difference across all the recorded sites in these two bands exhibited a linear relationship with corresponding group-level pair-wise rsfMRI connectivity measured in both DREADD and control animals during the CNO active window (Fig. $3 L, \delta R^{2}=0.92, p=0.002$; slow $R^{2}=0.73, p=0.029$, uncorrected). Only $\delta$ LFP coupling however retained a significant correlation with rsfMRI connectivity upon FDR correction $(q=0.008, F D R$ corrected). A much weaker correlation between band specific LFP coherence and pairwise rsfMRI connectivity was apparent for all the other bands (Fig. $3 \mathrm{~L}, \mathrm{p}>0.11$ all bands). These findings were paralleled by the observation of similarly robust increased interareal $\delta$ - and slow-band LFP phase coherence, a widely used measure of functional synchronization in LFP/EEG studies ${ }^{42}$ (Fig. 3M). Taken together, these results corroborate a neural origin for our imaging findings and implicate increased slow and $\delta$ LFP coherence as a plausible neural driver of the observed rsfMRI oversynchronization.

A

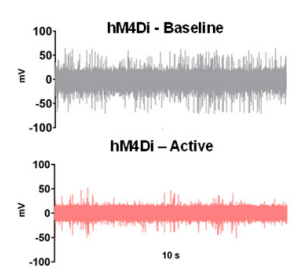

D

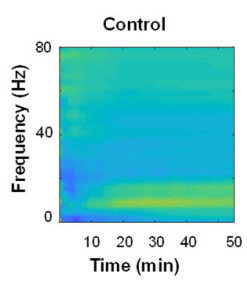

F

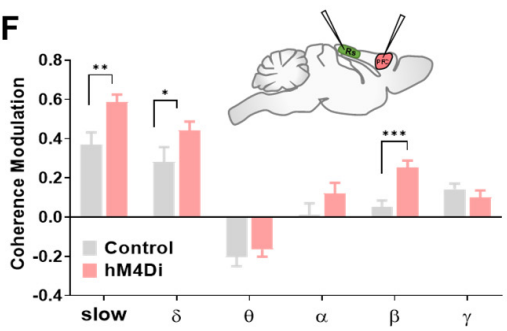

I

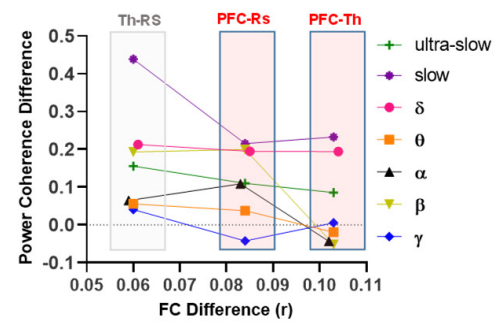

hM4Di
B
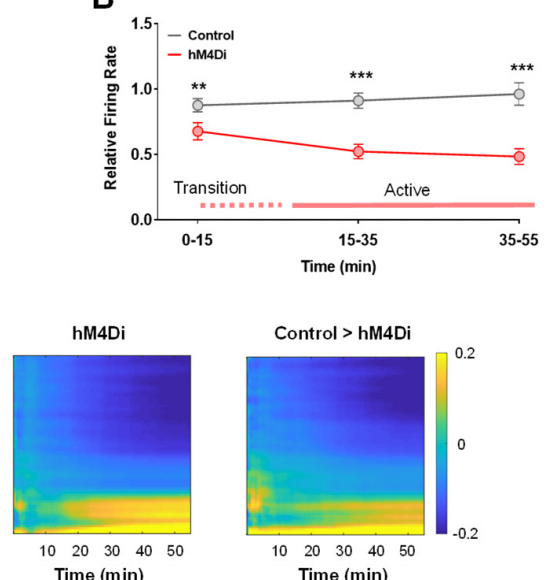

G

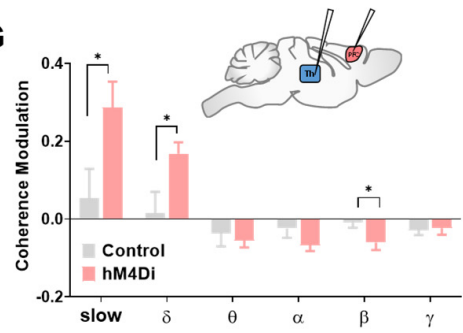

L

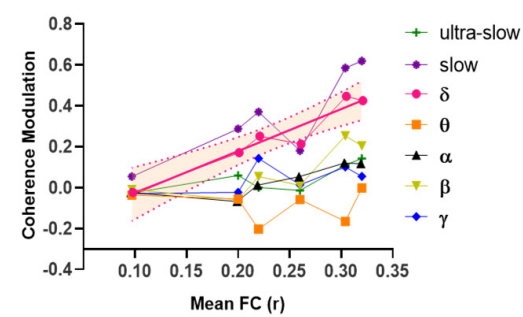

C
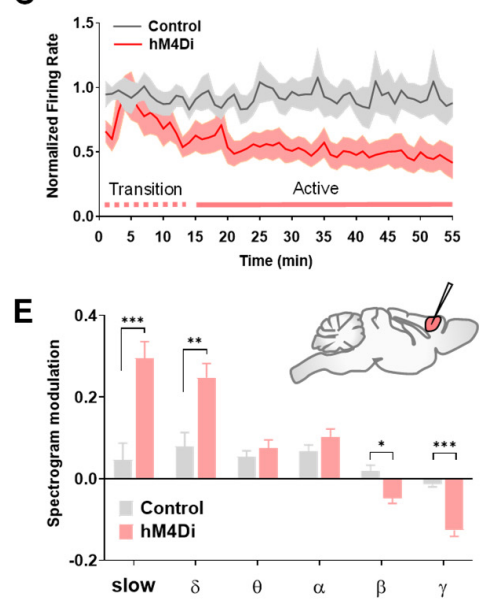

H

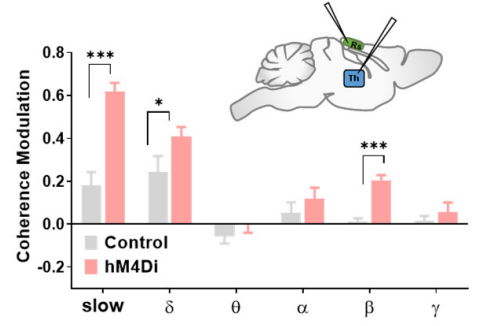

M

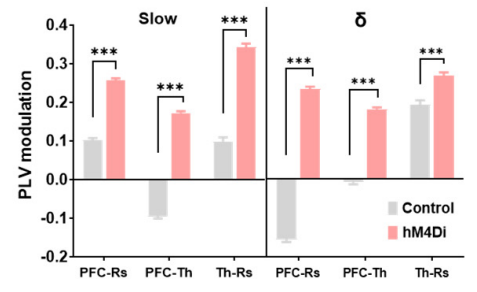




\section{Figure 4}

Chemogenetic silencing of the PFC results in decreased $y$ activity and increased interareal slow oscillatory coherence. (A) Representative raw traces collected before and after CNO injection in a representative recordings site of hM4Di animal. (B-C) Reduced firing rate in hM4Di-expressing mice $(n=5)$ compared to GFP-transduced controls $(n=5)$. (D) Mean post-injection spectrogram in control (Left), hM4Di-expressing animals (center), and mean between group difference (right). (E) Quantification of band-specific power spectrum changes upon CNO injection in both groups (F-G-H). Baseline normalized power coherence at different frequency bands for PFC-Retrosplenial (Left), PFCthalamus and Retrosplenial-thalamus electrode pairs (mean $\pm S E M ; F D R$ corrected ${ }^{*} q<0.05, * * q<0.01$, $* * * q<0.001, t$ test). (I) Band specific coherence and mean functional connectivity (FC) difference (hM4Di-Control) for all pairs of electrophysiologically-probed regions. Mean FC data were extracted for corresponding regional pairs (Fig. 2) during the CNO active time window in hM4Di and control animals. (L) Correlation between corresponding band specific coherence and mean functional connectivity for all pairs of electrophysiologically-probed regions (PFC-Rs; PFC-Th; Rs-Th). Shaded area indicates $95 \% \mathrm{Cl}$ for $\delta$. (M) Baseline normalized phase coherence in slow and delta band between electrode pairs (mean \pm SEM; ${ }^{*} p<0.05,{ }^{* *} p<0.01,{ }^{* *} p<0.001, t$ test, FDR corrected). 


\section{Discussion}

Here we combine neural perturbations and mouse rsfMRI to investigate how rsfMRI topography reconfigures in response to targeted cortical inactivation. We report that chronic and acute neural silencing of the mouse PFC can counterintuitively increase rsfMRI connectivity with DMN terminals directly innervated by the silenced area. Further electrophysiological investigations revealed that chemogenetic silencing of the PFC is associated with increased interareal LFP coherence, arguing against a purely vascular origin for the observed hyper-connectivity, and implicating low-frequency neural processes in the establishment of the observed rsfMRI overconnectivity.

While critically shaped and constrained by underlying axonal connectivity ${ }^{2-4}$, spatiotemporal correlations in spontaneous rsfMRI activity can dynamically reconfigure in response to local perturbations. In-depth investigations of the reconfiguration patterns resulting from regional suppression of neural activity are of special interest, as these allow for a targeted deconstruction of rsfMRI coupling and may offer opportunities to mechanistically interpret aberrant rsfMRI connectivity patterns in neurological conditions characterized by loss of cortical function. ${ }^{8,10,43}$. Leveraging the recent implementations of chemo-fMRI in the mouse ${ }^{21}$, we causally probed how cortical silencing affects brain-wide rsfMRI coupling. In contrast to theoretical ${ }^{10}$ and experimental ${ }^{8}$ evidence predicting that regional inactivation of a neural node would result in reduced functional synchronization with its direct anatomical targets, we found that both chronic and acute silencing of the PFC can counterintuitively increase rsfMRI connectivity within thalamo-cortical substrates of the mouse DMN, encompassing cortical and subcortical regions densely innervated by inactivated medial prefrontal areas.

Our results advance our understanding of the principles underlying brain-wide rsfMRI coupling in two directions. First, we provide causal evidence that regional suppressions of brain activity does not necessarily lead to reduced neural and functional coupling between the inactivated area and its direct anatomical projection targets, but can result in increased rsfMRI connectivity via enhanced, less-localized slow oscillatory coherence. These observations point at a highly dynamic and nonmonotonic relationship between structural and functional connectivity, underscoring a critical contribution of remote sources of large-scale neural covariation (e.g. $\delta$ and slow oscillations ${ }^{35,44}$ ) to the establishment of interareal rsfMRI coupling. This view is in keeping with correlational evidence of a dissociation between rsfMRI connectivity and underlying anatomical connections, such in the case of acallosal brains in which preserved bi-hemispheric connectivity has been repeatedly observed 7,15 , 
16. It should be emphasized here that these results should not be intended as a refutation of the strong structural foundations of rsfMRI connectivity, but rather the basis of an updated model in which in which reciprocal interareal rsfMRI coupling can be strongly biased, or even overridden, by slow synchronized input from global rhythm generators.

Second, our results also provide a novel reference framework for the interpretation and reverse engineering of rsfMRI overconnectivity observed in brain pathology, especially in degenerative or neurological states characterized by rsfMRI oversynchronization. Although sometimes compounded by pathophysiological rearrangements in synaptic activity ${ }^{45}$, observations of unexpected increases in functional connectivity in neurological conditions characterized by loss of cortical function such as Alzheimer's disease or stroke have been speculatively conceptualized as the result of compensatory rerouting of signal propagation along indirect structural paths, a neuroadaptive strategy aimed at maintaining task performance ${ }^{19,20,46}$. Our findings offer an alternative, network-level mechanism for these clinical observations, suggesting that rsfMRI overconnectivity may reflect an imbalance between direct high frequency interareal synchronization due to direct axonal communication and low-frequency synchronization mediated by single subcortical centers such as the polymodal thalamus ${ }^{11}$ or global modulatory input 47,48 , a conceivable scenario in early-stage degenerative or neurological states characterized by loss of neuronal function in higher order cortical areas. EEG and MEG studies in patients with stroke or neurodegenerative brain disorders lend indirect support to this hypothesis, as both rsfMRI overconnectivity and robust $\delta$ hyper-synchronization have been observed ipsilaterally to lesioned areas in stroke ${ }^{20,49}$. Similar findings have been reported in Alzheimer's disease patients, in which this effect appears to be especially prominent in polymodal cortical areas 50, 51. Within such a framework, functional impairments in cortical activity following degenerative pathology could manifest as hyper-synchronization during pre-disease states, eventually reverting to hypotrophy-associated under-connectivity at advanced stages of brain pathology.

While a neural mass phenomenon like interareal rsfMRI coupling cannot be mechanistically dissected into discrete circuital elements, our observation of foci of over-connectivity in polymodal thalamic areas and the oversynchronization of these with larger cortical territories is intriguing, as it points at a putative involvement of higher order thalamic relay in the generation (or propagation) of the observed overconnectivity. This hypothesis would be consistent with the observation that full expression of $\delta$ and slow oscillatory activity requires thalamic participation ${ }^{52,53}$, and with recent 
evidence pointing at a the presence of a prefrontal-thalamic loop involved in the generation and propagation of $\delta$ synchronization ${ }^{11}$. However, other modulatory mechanisms ${ }^{12,47}$ or subcortical substrates ${ }^{54}$ may similarly (or alternatively) play a role in the observed oversynchronization, and the identification of neural generators of rsfMRI coupling and their involvement in the reconfiguration of rsfMRI topography in response to local perturbations remain an open issue.

The observed increases in slow and $\delta$ band coherence upon silencing of the PFC is in excellent agreement with previous reports of a robust association between spontaneous fMRI activity and slow oscillatory activity $35,41,44,55-58$. Our findings corroborate and expand these investigations by showing that reconfiguration of rsfMRI connectivity in response to local cortical inactivation is supported by increased interareal slow oscillatory coherence. Recent work has linked ultra-slow ( $\sim .1 \mathrm{~Hz})$ variation in gamma-band amplitude to the vasomotor activity underlying intrinsic rsfMRI connectivity ${ }^{36,40,}$ suggesting that this rhythm may serve as a possible primary generator of interareal rsfMRI coupling. Interestingly, while we found evidence of ultra-slow $\curlyvee$ envelope coherence across functionally connected areas under basal conditions (Fig. S7), the corresponding coherence increases upon chemogenetic manipulations were very small and did not correlate with corresponding pairwise rsfMRI overconnectivity. This finding suggests that, under the conditions of our manipulations, rsfMRI over-connectivity is most plausibly explained by increased canonical band (e.g. $\delta$ ) low-frequency inter-areal coherence. While the exact significance of this uncoupling remains to be determined, the greatly reduced $\curlyvee$ power produced by the employed chemogenetic silencing could conceivably have led to a dissociation between coherence in canonical bands and ultra-slow variations in $\Upsilon$ envelope, with the ensuing rsfMRI connectivity being dominated by canonical low-frequency bands LFP coherence. Additional investigations leveraging concurrent hemodynamic and electrophysiological recordings e.g. via optical imaging ${ }^{40}$ may help disambiguate and clarify the significance of these observations.

Given the highly dynamic nature of rsfMRI network activity, it is conceivable that the sign and neurophysiological signatures identified in this study might be affected by brain state and arousal levels. Although the light sedation protocol used in our measurements enhances slow oscillatory rhythms ${ }^{59}$, such a brain state is unlikely to be the primary reason for our observation of rsfMRI overconnectivity, as similarly increased rsfMRI connectivity between homotopic (i.e. reciprocally connected) cortical regions has been reported for example upon inhibitory transcranial magnetic stimulation (TMS) of the primary motor cortex in awake humans ${ }^{60}$. Analogous findings have been 
measured in conscious humans using electro encephalographic recordings ${ }^{61}$. These results are of interest as they may support a possible generalization of our findings to other, non-associative cortical districts. However, two studies reported weak rsfMRI suppression when inhibitory chemogenetics was applied in the postero-dorsal cingulate cortex in anesthetized mice and restrained rats, respectively ${ }^{62,63}$. While the lack of electrophysiological recordings in these rodent studies prevent a direct comparison with the results we report here, these observations seem to suggest instead that the reconfiguration patterns in response to cortical silencing could be strictly area specific. Interestingly, the same TMS study from Watanabe and colleagues mentioned above ${ }^{60}$ also showed that excitatory pulsed TMS does result in lower rsfMRI synchronization, pointing at a bidirectional relationship between cortical activity and interareal rsfMRI coupling. An analogous bidirectional relationship might also be present in the mouse because, mirroring our results, decreased rsfMRI connectivity has been recently described with excitatory DREADD stimulations of the mouse somato-motor areas using a sedation protocol similar to the one employed here ${ }^{64}$. Additional systematic investigations are warranted to clarify whether and how the anatomical location and physiological direction of cortical manipulation differentially affect rsfMRI functional connectivity as a function of brain state.

In conclusion, the present work provides causal evidence that silencing of a cortical region can lead to counter-intuitively increased rsfMRI connectivity between the inactivated region and its targets, and that this seemingly paradoxical functional overconnectivity may result from increased interareal low-frequency neural coherence. These findings point at a non-monotonic relationship between regional cortical activity and network-wide rsfMRI connectivity and define novel testable network-level mechanisms for the interpretation of rsfMRI overconnectivity in clinical conditions characterized by loss of cortical function. 


\section{Acknowledgements}

This work has received funding from the European Research Council (ERC) under the European Union's Horizon 2020 research and innovation program (\#DISCONN; no. 802371 to A.Go.), the Brain and Behavior Foundation (NARSAD; Independent Investigator Grant; no. 25861), Simons Foundation (602849 to S.P) and NIH Brain Initiative U19 NS107464 (S.P). A.Go. also acknowledges support by the Simons Foundation (SFARI 400101), the NIH (1R21MH116473-01A1) and the Telethon foundation (GGP19177). We thank Michael Lombardo, Elizabeth De Guzman and all the members of the Gozzi laboratory for critically reading the manuscript. We also thank Thomas Gozzi for figure renderings.

\section{Author contribution}

A.Go. conceived and supervised the entire study. AG and SP designed and supervised electrophysiological analyses. CC carried out viral transduction and the fMRI experiments with help from A.Ga. FR designed, carried out and analyzed electrophysiological recordings. GI, SP, SV, NS, FR, CC, MP, DGB and A.Go. analyzed rsfMRI and electrophysiological data. A.Go. and S.P. secured funding. A.G. wrote the manuscript with contributions from S.P. 


\section{Materials and Methods}

Ethical statement. All in vivo experiments were conducted in accordance with the Italian law (DL 26/214, EU 63/2010, Ministero della Sanità, Roma). Animal research protocols were reviewed and consented by the animal care committee of the University of Trento and Italian Ministry of Health (authorization no. 852/17 to A.G.). All surgical procedures were performed under anesthesia.

Animals. Adult (6 week old) male C57BI6/J were purchased from Jackson Laboratories (Bar Harbor, ME, USA). Mice were housed with temperature maintained at $21 \pm 1^{\circ} \mathrm{C}$ and humidity at $60 \pm 10 \%$.

Anatomical definition of mouse medial prefrontal cortex. Our anatomical definition of mouse medial prefrontal cortex (PFC) reflects recent neuroanatomical 65 and cytoarchitectural cross-species comparisons ${ }^{66}$, according to which the mouse PFC comprises a prelimbic region, corresponding to primate Brodmann area 32 (A32), the anterior cingulate cortex, corresponding to Brodmann area A24b, and the infralimbic cortex, corresponding to Brodmann area A24a. Our viral manipulations were therefore aimed to silence an anatomical ensemble comprising all the above mentioned regions at the following coordinates, expressed in millimeter from Bregma: 1.7 from anterior to posterior, +/- 0.3 lateral, -1.7 deep ${ }^{67}$.

Viral injections: Mice were anesthetized with isoflurane and head-fixed in a stereotaxic apparatus (Stoelting). Injections were performed with a Hamilton syringe mounted on Nanoliter Syringe Pump with controller (KD Scientific), at a speed of $0.05 \mu \mathrm{l} / \mathrm{min}$, followed by a 5-10 min waiting period, to avoid backflow of viral solution. The following injections volumes were employed: $300 \mathrm{~nL}$ (AAV8hSyn-hM4D(Gi)-mCherry and AAV8-hSyn-GFP; http://www.addgene.org) or 2 $\mu \mathrm{L}$ (AAV8-hSyn-MYCmKir2.1(E224G/Y242F)-IRES-GFP, 24, http://www.vectorbiolabs.com or AAV8-hSyn-GFP; http://www.addgene.org) of viral suspension were injected bilaterally in the mouse PFC (see coordinates above). rsfMRI or electrophysiological recordings were carried out no sooner than three weeks after the injection, to allow for maximal viral expression.

\section{rsfMRI acquisitions}

The animal preparation protocol was recently described in detail 13,68, 69. Briefly, mice were anesthetized with isoflurane ( $5 \%$, induction), intubated and artificially ventilated ( $2 \%$, surgery). The left femoral artery was cannulated for continuous blood pressure monitoring. At the end of surgery, isoflurane was discontinued and substituted with a shallow halothane regimen (0.75\%) to obtain light 
sedation and to preserve cerebral blood flow auto-regulation ${ }^{70}$. Ventilation parameters were adjusted to maintain normo-physiological $\mathrm{p}_{\mathrm{a}} \mathrm{CO}_{2}(<40 \mathrm{mmHg})$ and $\mathrm{p}_{\mathrm{a}} \mathrm{O}_{2}$ levels $(>90 \mathrm{mmHg}$, corresponding to $>98 \%$ hemoglobin saturation).

rsfMRI data acquisition commenced 30 min after isoflurane cessation. Functional images were acquired with a $7 \mathrm{~T}$ MRI scanner (Bruker, Biospin) as previously described ${ }^{71}$, using a $72 \mathrm{~mm}$ birdcage transmit coil and a 4-channel solenoid coil for signal reception. Single-shot BOLD rsfMRI time series were acquired using an EPI sequence with the following parameters: TR/TE 1000/15 ms, flip angle 60', matrix $98 \times 98$, FOV $2.3 \times 2.3 \mathrm{~cm}, 18$ coronal slices, slice thickness $550 \mu \mathrm{m}$.

rsfMRI acquisition with Kir2.1-transduced (AAV8-hSyn-MYC-mKir2.1(E224G/Y242F)-IRES-GFP, n= 16) and control mice (AAV8-hSyn-GFP, $n=19$ ) encompassed 35-minute long timeseries, corresponding to 2100 volumes.

Chemo-fMRI acquisitions comprised two consecutive rsfMRI timeseries, encompassing 1800 volumes (30 min) and 2100 volumes (35 min), respectively. CNO (2 mg/kg, Sigma Aldrich) was injected intravenously fifteen minutes (volume \#900) after the start of the first scan. The first $900 \mathrm{fMRI}$ volumes of this first timeseries scan were used as pre-CNO baseline rsfMRI reference in time-resolved analyses. Based on the pharmacokinetic profile of CNO, the post CNO window was split into temporal domains as follows: the first 15 min post injection (900 volumes) were considered part of a drug equilibration window, while the following 35 min (2100 volumes) were considered to cover the DREADD active time window ${ }^{30}$. All group comparisons in the chemo-fMRI study were carried out within this latter time window, unless otherwise stated. After post-mortem analyses of viral expressions, a total of $\mathrm{N}=15 \mathrm{hM} 4 \mathrm{Di}$ and N=19 GFP-transduced animals were retained for analyses.

\section{Image preprocessing and analysis}

Raw rsfMRI timeseries were preprocessed as described in previous work ${ }^{13,68}$. Briefly, the initial 120 volumes of the time series were removed to allow for gradient equilibration effects. Data were then despiked, motion corrected and spatially registered to a common reference template. Motion traces of head realignment parameters ( 3 translations +3 rotations) and mean ventricular signal (corresponding to the averaged BOLD signal within a reference ventricular mask) were used as nuisance covariates and regressed out from each time course. All rsfMRI time series also underwent band-pass filtering within a frequency window of $0.01-0.1 \mathrm{~Hz}$ and spatial smoothing with a full width at half maximum of $0.6 \mathrm{~mm}$. To control for the effects of global fMRI signal regression on the mapped 
changes, all rsfMRI timeseries were also recomputed by regressing average fMRI signal within an intracerebral mask

rsfMRI connectivity of the mouse DMN in Kir2.1 and chemo-fMRI scans was probed using a seed-based approach. In the case of the chemo-fMRI study, this quantification was carried out during the CNO active time window. A $5 \times 5 \times 2$ seed region was selected to cover the PFC areas targeted by viral injections. Voxel-wise intergroup differences in seed-based mapping were assessed using a 2tailed Student's t-test $(|\mathrm{t}|>2, \mathrm{p}<0.05)$ and family-wise error (FWE) cluster-corrected using a cluster threshold of $p=0.05$ as implemented in FSL (https://fsl.fmrib.ox.ac.uk/fsl/). The antero-posterior connectivity profile of the DMN was assessed by computing Person correlation between the PFC seed abovementioned and a series of $6 \times 6 \times 2$ voxel seeds placed along the midline extension of the cingulate and retrosplenial cortices as previously described ${ }^{27}$. Quantification of cortico-thalamic connectivity was carried out with respect to a meta-regional parcellation of the mouse cortex in volumes-of-interest. To rule out a possible confounding contribution of spurious neurovascular changes in CNO-induced rsfMRI connectivity alterations, we calculated and statistically compared the characteristic hemodynamic response function between kir2.1 and control mice, and between hM4Di-expressing and control mice upon CNO-administration (active phase), as previously described 27,72 .

Whole-brain connectivity in hM4Di and Control mice was calculated across a set of volumes of interest recapitulating anatomical areas of the Allen brain atlas. The anatomical probed area were selected according to their coverage of previously characterized network systems of the mouse brain 22, 68, 71: TH: thalamus (thalamus polymodal association cortex related, Thalamus Sensory-Motor cortex related); STR: striatum (Striatum dorsal region left, striatum dorsal region right, striatum ventral region left, striatum ventral region right); LCN: lateral cortical network (LCN: primary motor cortex left, primary motor cortex right, primary somatosensory cortex left, primary somatosensory cortex right, secondary somatosensory cortex left, secondary somatosensory cortex right, Lateral septal complex left, lateral septal complex right); HCP: hippocampus (Ammon's horn left; Ammon's horn right, dentate gyrus left; dentate gyrus right, Entorhinal area left, entorhinal area right, subiculum left, subiculum right); DMN: default mode network (anterior cingulate area; Infralimbic area, secondary motor cortex left, secondary motor cortex right, orbital area, prelimbic area, posterior parietal association areas left, Posterior parietal association areas right, retrosplenial area); 
To relate the strength of underlying anatomical connectivity to the regions exhibiting increased rsfMRI connectivity with voxel resolution we extracted outgoing projections from the affected PFC regions using a spatially-resampled $\left(0.027 \mathrm{~mm}^{3}\right)$ version of a voxel scale model of the Allen Brain Institute structural connectome ${ }^{4}$. We then plotted the strength of PFC-departing structural projections against the corresponding between-group difference in rsfMRI connectivity using the cluster-corrected difference map, and assessed differences in the distribution of overconnected areas with respect to all the brain voxels using a Wilcoxon rank-sum test.

To quantify the contribution of distinct thalamic subregions to overall group differences, we used k-means clustering to partition voxels within the thalamus, based on whole-brain rsfMRI groupdifference obtained using the PFC seed as recently described (Arthur and Vassilvitskii, 2007; Schleifer et al, 2019). This approach revealed two major thalamic clusters, one medial and one bilateral partition encompassing sensory areas. Seed-based functional connectivity was subsequently computed for each of the two-resultant k-means clusters independently and the resulting functional connectivity maps compared and quantified across cortical VOIs.

Electrophysiological recordings. Electrophysiological recordings were carried out in animals subjected to the same animal preparation and sedation regime employed for rsfMRI mapping (Ferrari et al, 2012; Sforazzini et al, 2014). Briefly, mice were anaesthetized with isoflurane (5\% induction), intubated, artificially ventilated ( $2 \%$ maintenance), and head-fixed in a stereotaxic apparatus (Stoelting). The tail vein was cannulated for CNO injection. To ensure maximal consistency between viral injections and recording site, the skull surface was exposed and an insertion hole in the right PFC was gently drilled through the skull corresponding to the location of prior viral injection point. A single shank electrode (Neuronexus, USA, interelectrode spacing $1-2.5 \mathrm{~mm}$ ) was next inserted through the overlying dura mater by a microdrive array system (Kopf Instruments, Germany) at an insertion rate of $1 \mu \mathrm{m} / \mathrm{min}$ to reach the same stereotaxic coordinates employed for viral injection.

The site receptive fields were plotted manually and the position and size of each field were stored together with the acquisition data. After electrode insertion, isoflurane was discontinued and replaced by halothane at a maintenance level of $0.75 \%$, to induce rsfMRI-comparable sedation. Electrophysiological data acquisition commenced 1 hour after isoflurane cessation. Such transition 
time was required to ensure complete washout of isoflurane anesthesia and avoid residual burstsuppressing activity associated with extended exposure to deep anesthetic levels.

Neural activity was next recorded in consecutive 5 min time bins to cover a 15 min preinjection time window, and a 60 min post CNO time-frame in $n=5$ hM4Di and $n=5$ GFP-expressing mice. Signals were amplified using an RHD 2000 amplifier system (Intan Technologies) at a sampling rate of $20 \mathrm{kHz}$. In the case of control Kir2.1 recordings, a four shank electrode was inserted along the coronal plane to bi-hemispherically cover the right (Kir2.1-expressing) and left (GFP-expressing) PFC $(n=4)$. The left region served as internal reference control to better assess the efficacy of Kir2.1 silencing. Electrophysiological signals were then recorded into 5 min time bins to cover a 35 min timewindow.

To measure multi-electrode coherence, three electrodes were inserted in key cortical and subcortical substrates identified as overconnected in our chemo-fMRI mapping. A multi-probe micromanipulator (New-Scale Technologies) was used to insert a 32 channels four shank electrode (Neuronexus, USA, interelectrode spacing $1-2.5 \mathrm{~mm}$ ) in the right prefrontal cortex, and two 16 channels single shank electrode (Neuronexus, USA, interelectrode spacing 1 - $2.5 \mathrm{~mm}$ ) in the right centromedial thalamus and retrosplenial cortex, respectively. To reduce tissue damage, an insertion rate $<1 \mu \mathrm{m} / \mathrm{min}$ was employed, allowing for a 30 minute equilibration every $400 \mu \mathrm{m}$ traveled. Ground electrodes were put in contact with the cerebral brain fluid through a window drilled in the skull. Signals were then recorded into $1 \mathrm{~min}$ time bins to cover a 15-min pre-injection baseline and a 40-min post CNO time window in $n=5$ hM4Di and $n=5$ GFP-expressing adult C57BI6/J mice.

LFP and multi-unit activity (MUA). To compute the LFP signal, raw extracellular recordings were first down-sampled to $4 \mathrm{kHz}$, then band-pass filtered to 1-250 Hz using a previously published two-step procedure ${ }^{73}$. Briefly, raw timeseries were first low-pass filtered using a $4^{\text {th }}$ order Butterworth filter with cut-off frequency of $1 \mathrm{kHz}$. The resulting timeseries were next down-sampled to $2 \mathrm{kHz}$, then again filtered using a Kaiser window filter between $1 \mathrm{~Hz}$ to $250 \mathrm{~Hz}$ (with a sharp transition bandwidth of $1 \mathrm{~Hz}$, passband ripple of $0.01 \mathrm{~dB}$ and a stop band attenuation of $60 \mathrm{~dB}$ ) and then resampled at 1 $\mathrm{kHz}$. All the filtering was applied both in forward and backward temporal direction to avoid any phase transitions due to filtering.

To compute multi-unity activity (MUA) we again followed the procedure described in ${ }^{73}$. Briefly, we computed a band-passed high-frequency signal using a $4^{\text {th }}$ order Butterworth filter with $a<100 \mathrm{~Hz}$ 
cut off frequency, then band-pass filtered between 400 and $3000 \mathrm{~Hz}$ using a Kaiser window filter (with transition band of $50 \mathrm{~Hz}$, stopband attenuation of $60 \mathrm{~dB}$, and passband ripple of $0.01 \mathrm{~dB}$ ). From the high frequency signal we detected spike times using a spike detection threshold corresponding to 4-times the median of the high frequency signal, then divided by 0.6745 as suggested in (Quiroga et al, 2004). Spikes were considered to be biologically plausible, and as such retained in these computations, only if occurring more than $1 \mathrm{~ms}$ apart. This method does not permit the isolation of single units. For the purpose of this study, however, recording the activity of a small group of neurons provided a sufficient (if not better) estimate of local spike rate changes.

To quantify effectiveness of Kir2.1 in suppressing spontaneous activity, firing rate was computed (in units of spikes/s) by dividing the number of spikes per electrode by the recording duration in seconds. The resulting spike rates were averaged across the channels corresponding to the virally targeted or control region. The average spike rate for four subjects were next tested against each other using paired student $t$ test. To assess the effect of chemo-fMRI manipulations, spiking activity was computed in hM4Di and GFP-expressing mice as described above and segmented into one-minute bins. We next computed the channel averaged firing rate for each segment, and normalized this value with respect to the firing rate recorded during baseline (pre-CNO) period. The resulting baseline normalized firing rate index was then used to assess changes in spiking rate upon CNO injection in the two experimental cohorts.

To determine the time lag at which different firing rate samples from the same channels could be considered as approximately statistically independent we computed for each subject the autocorrelation of the channel-averaged firing rate for each subject and computed the time lag after which the autocorrelation function drops below the $95^{\text {th }}$ percentile. We then, for each subject, retained samples of the baseline-normalized firing rate at different times separated by the above obtained lags. We next separately analyzed the baseline-normalized rates in three different periods: 0 to 15 minutes (transient time), 15 to 35 minutes and 35 to 55 minutes after CNO the injection (active times). We pooled all the retained data points in these windows both over time and over subjects and then compared the median between the two populations using two-sided Wilcoxon rank-sum test. The obtained p-values were corrected using a Bonferroni-Holm correction.

LFP spectrograms were computed using a Fourier transform with a Kaiser window with a frequency resolution of $0.15 \mathrm{~Hz}$, temporal resolution of 6 seconds, and with 50\% overlapping windows. 
Spectrograms and their differences were smoothed in time with the resolution of 30 seconds, and in frequency with the resolution of $1 \mathrm{~Hz}$ using median filter. To quantify the effect of CNO on LFP rhythms, we computed a spectrogram modulation index as follows. First, we computed the channelaveraged spectrograms for the duration of the baseline recording. Next, we averaged time-frequency spectral profiles over time, resulting in frequency-resolved spectral profiles. The effect of CNO was next assessed by computing a modulation index, defined as the ratio of channel-averaged spectrogram after injection minus baseline, time-averaged spectrogram and the sum of the same quantities, for every time window and for every frequency. This modulation index ranges between 1 and 1 , and describes the changes due to drug injection over time, for each frequency.

To obtain a statistical assessment of CNO effects across groups and bands, we computed the autocorrelation of spectrograms for every subject at every frequency, using (as for our assessment of firing rate) only spectrograms computed at time points far enough to be approximately statistically independent. The time lag to determine such approximately independent points was identified as the interval after which the spectrum's autocorrelation function dropped below the $95^{\text {th }}$ percentile. We next computed the median of the modulation index over different frequency bands defined as follows: slow $(0.1-1 \mathrm{~Hz}), \delta(0.1-4 \mathrm{~Hz}), \theta(4-8 \mathrm{~Hz}), \alpha(8-12 \mathrm{~Hz}), \beta(12-30 \mathrm{~Hz})$ and $y(30-70 \mathrm{~Hz})$. Data within each band were pooled over uncorrelated time points (determined as above by taking time samples separated by lags at which autocorrelation became negligible) and over subjects, and the population medians were compared using two-sided Wilcoxon rank-sum tests, followed by Bonferroni-Holm correction.

\section{Multielectrode coherence}

Channel averaged spectrograms were preprocessed as described above. Raw spectral power coherence was assessed by computing the magnitude of squared coherency using Welch's overlapped averaged periodogram method ${ }^{74}$ with a $50 \%$ overlapping window of 2 seconds length. Coherence was calculated for every 60-second bin of channel-averaged recordings. Temporal smoothing was carried out using a 60-second median filter as described above. CNO spectrograms (30-40 post CNO injection window), were normalized with respect to the last 3 minutes of pre-CNO baseline using a modulation index as before. To obtain a quantifiable assessment of the CNO effect across different groups and bands, data within each band were pooled into 60-second bins, and subject and the population medians were compared for each region separately using a two-sided 
Wilcoxon rank-sum tests, followed by Bonferroni-Holm correction. The use of 60 second bins was motivated by estimations of the time lags at which autocorrelation of electrophysiological signals drops below the $95^{\text {th }}$ percentile value (and thus samples are approximately independent) as described above.

To assess changes in ultra-slow fluctuations in the $\curlyvee$ band envelope of LFP, following the work from Nir et al. ${ }^{36}$, we computed LFP data spectrogram for the last 10 minutes of baseline and 30 to 40 minutes of post injection window, using a moving window of 2 seconds width and 0.75 percent overlap, resulting in estimation of the resulting ultra-slow power for each 0.5 second interval. The $\curlyvee$ band envelope was computed as the integrated power of the spectrograms in the $\curlyvee$ band $(30-70 \mathrm{~Hz})$, following previously published procedures ${ }^{40}$. The power spectrum of this time series was next computed, after correction for $1 / \mathrm{f}$ component ${ }^{40}$, revealing a peak in the $0.02-0.05 \mathrm{~Hz}$ region. Interareal coherence was then computed as described above for both experimental and control mice. To assess the effect of chemogenetic manipulations, we defined a coherence modulation index as described above for other frequency bands. Between group statistical assessments were carried out using two-sided Wilcoxon rank-sum tests, followed by Bonferroni-Holm correction.

To quantify interregional phase coupling, LFP data were first filtered using a third order Butterworth filter in delta band, and the instantaneous phase of each channel was computed by taking the phase of the analytical signal resulted from the Hilbert transform. For all possible pair of channels belonging to two different regions, we next computed the corresponding Phase Locking Value (PLV) as follows:

$$
\mathrm{PLV}=\frac{1}{\mathrm{~N}}\left|\sum_{j=1}^{N} e^{i\left(\theta_{c h 1}\left(t_{j}\right)-\theta_{c h 2}\left(t_{j}\right)\right)}\right|
$$

Where $\mathrm{N}$ is the number of data points in time and $\theta_{c h 1}\left(t_{j}\right), \theta_{c h 2}\left(t_{j}\right)$ are the instantaneous phase of the LFP of channel 1 and 2 at time j. The PLV value for each pair during the 30 to 40 minutes post CNO was next normalized with respect to the PLV value of the last 3 minutes of the pre-CNO baseline using a modulation index and was pooled over channel pairs and animals. The obtained population medians of the control and experimental group for each region pair were next compared using a twosided Wilcoxon rank-sum tests, followed by Bonferroni-Holm correction. 
Supplementary Figures

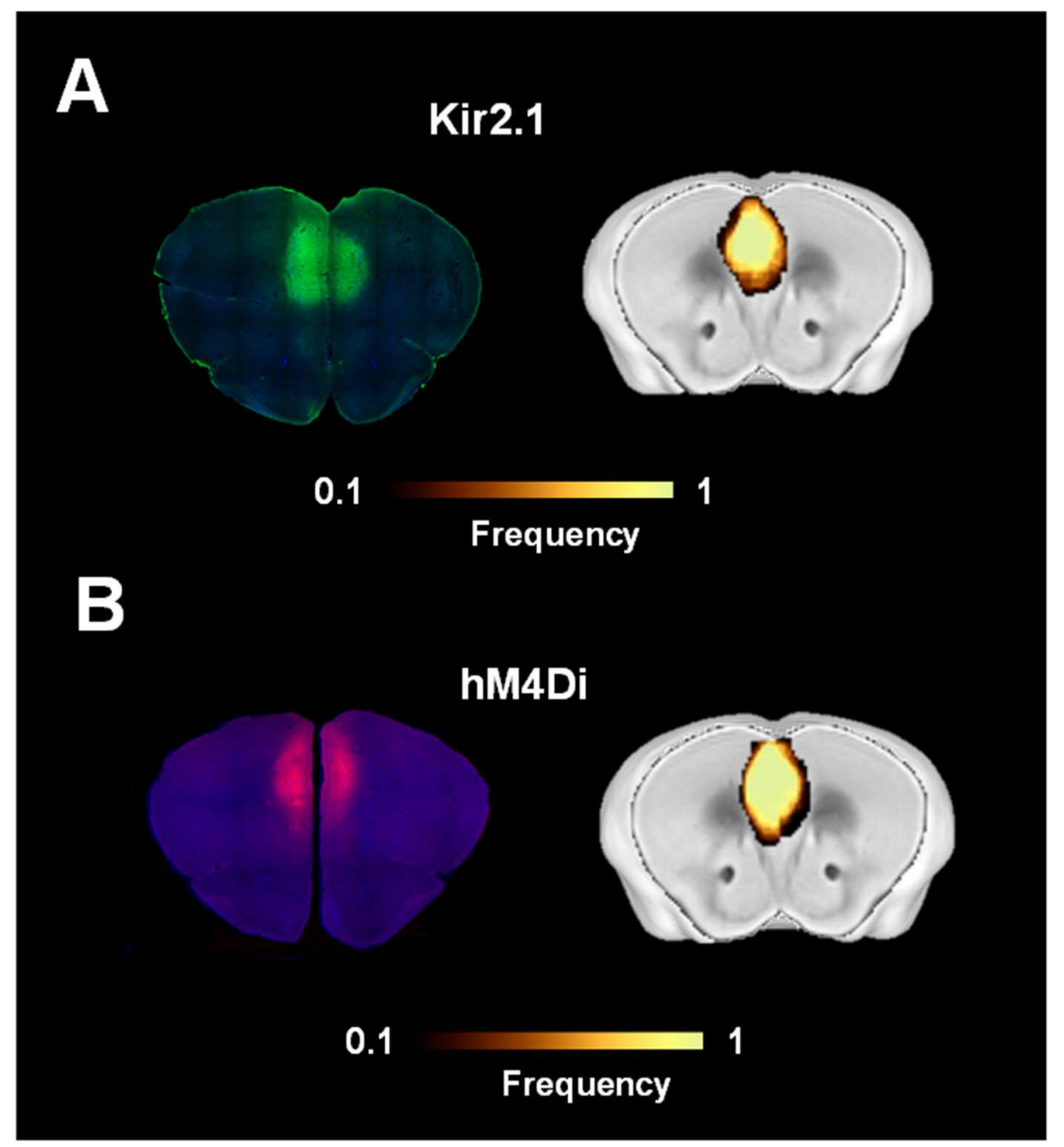

Figure S1. Viral expression localization. Brain sections showing (A) Kir2.1 (green) and (B) hM4Di (red) expression. Heat maps illustrate a qualitative regional assessment of viral expression across subjects. 
A

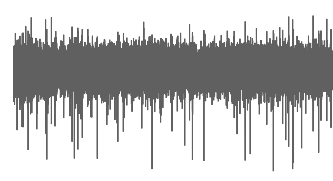

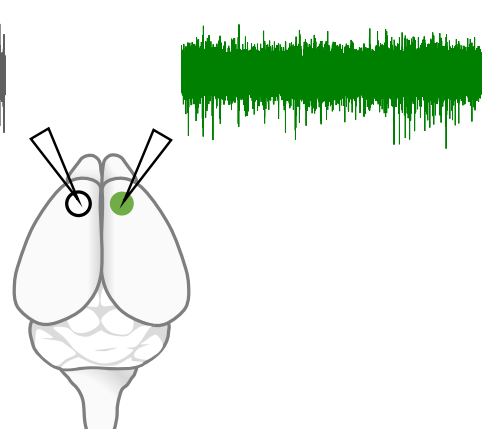

B

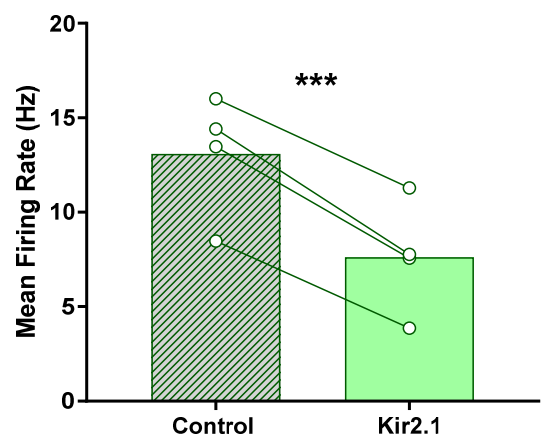

Figure S2. Overexpression of the potassium channel Kir2.1 in the PFC reduces spontaneous neural activity. (A) Experimental design: Kir2.1 injection was performed unilaterally in the right PFC. A viral vector encoding GFP was injected in the contralateral area. Electrophysiological recordings were carried out bilaterally using a four-shank electrode. (B) Mean spontaneous firing rate for the control side (no Kir2.1 expression), and the side expressing Kir2.1. ( $\left.n=4 ;{ }^{* *} p<0.005\right)$. 
A

Difference - Kir2.1 > Control
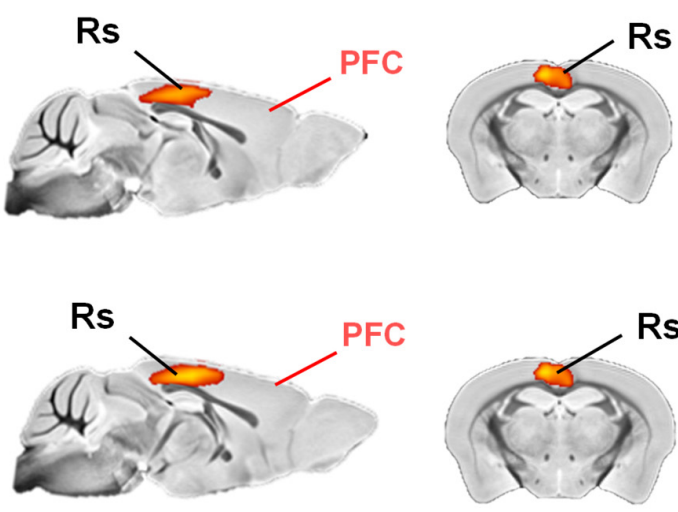

C

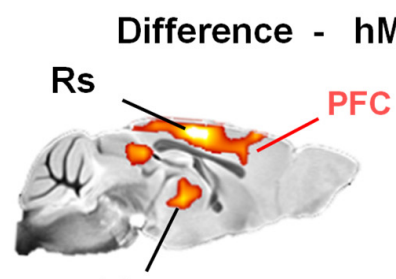

Th

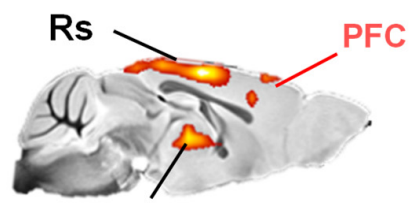

Th
Rs

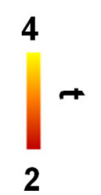

D

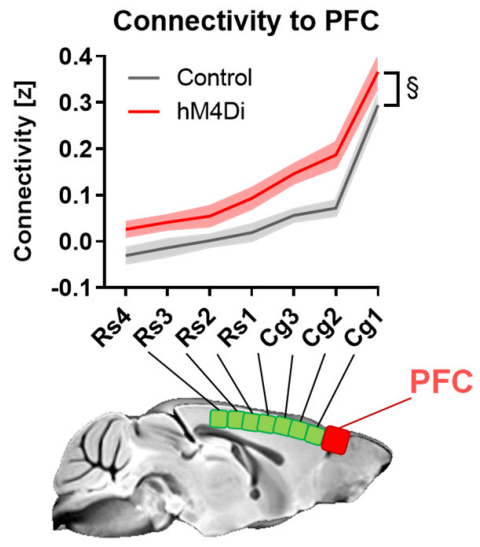

Figure S3. Foci of rsfMRI overconnectivity in Kir2.1 mice upon rsfMRI global signal regression (A) Between-group difference maps (Pearson's $r$, and corresponding T stat difference maps). (B) Anteroposterior profiling of rsfMRI connectivity of the PFC along the midline axis of the mouse DMN in the two cohorts revealing consistent overconnectivity in Kir2.1 mice. rsfMRI connectivity was here computed after fMRI global signal regressions. (C-D) rsfMRI overconnectivity in hM4Di mice is not affected by rsfMRI global signal regression (C) Between-group difference maps (Pearson's $r$, and corresponding T stat difference maps). (D) Antero-posterior profiling of rsfMRI connectivity of the PFC along the midline axis of the mouse DMN in the two cohorts upon fMRI global signal regressions. Data are plotted as mean \pm SEM. Cg: cingulate cortex; PFC: prefrontal cortex, RS: retrosplenial cortex; $\S p<0.05,2$-way ANOVA repeated measurements, genotype effect. 

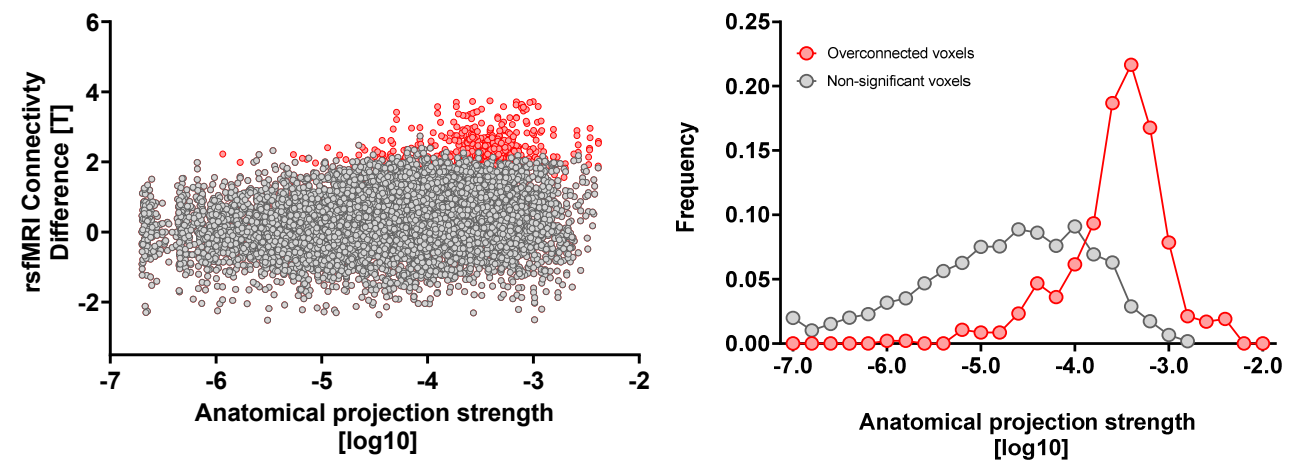

Figure S4. Voxels exhibiting rsfMRI overconnectivity upon chemogenetic silencing of the PFC are robustly innervated by the PFC. Left: scatter plot illustrating intergroup differences in rsfMRI connectivity as a function of PFC structural connectivity strength. Note that all significantly overconnected voxels (red) contain robust axonal projections from the PFC. Right: distribution of voxels exhibiting the most significant rsfMRI connectivity (red) and those that are not affected (grey). 

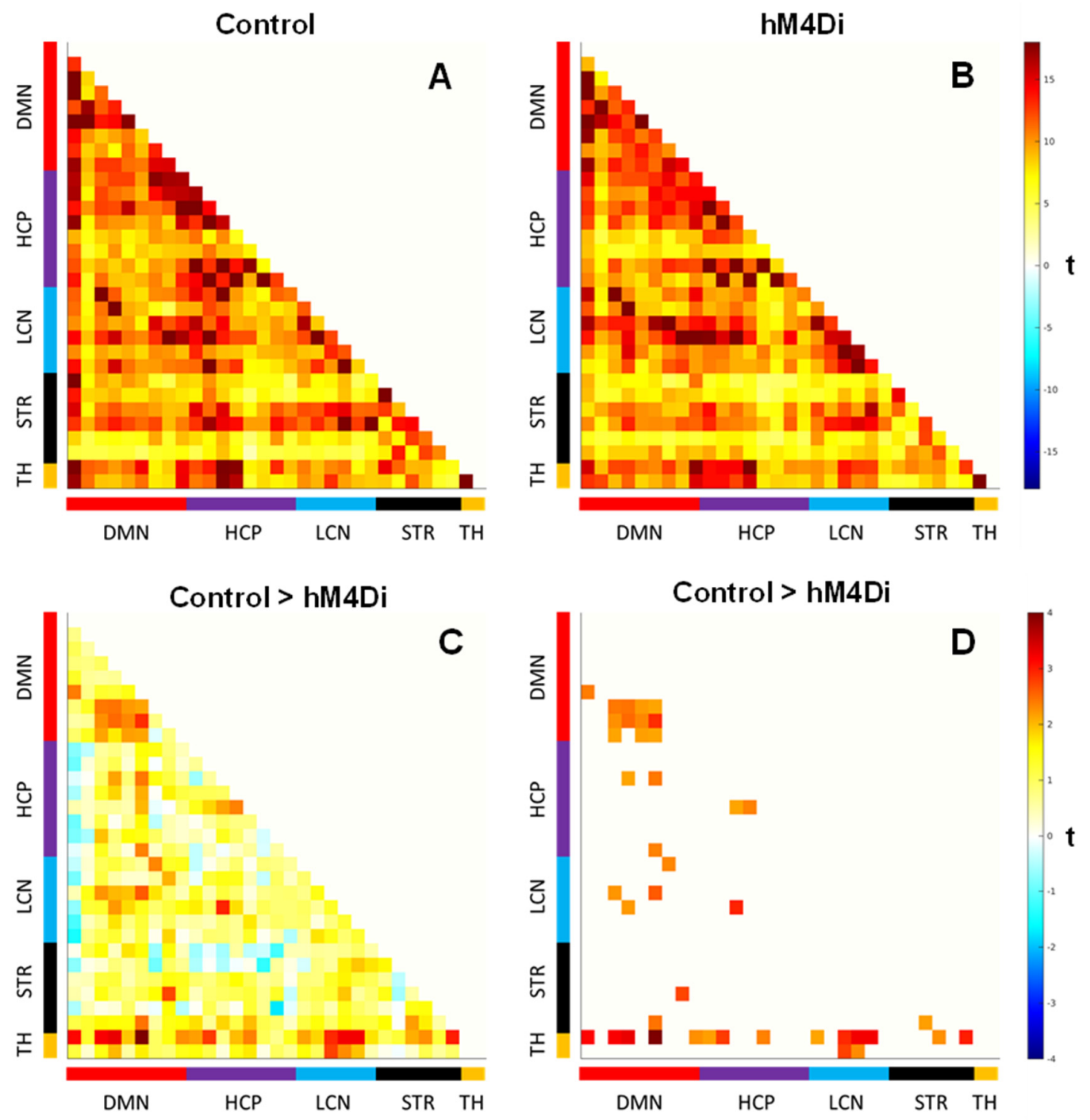

Figure S5. Whole-brain rsfMRI connectivity in control and HM4Di mice. Correlation matrices in (A) and (B) depict inter-areal connectivity in control and hM4Di mice respectively. (C) Mean difference map $(t)$, and $(D)$ regions exhibiting connectivity differences larger than $|t|>2.1$, corresponding to p<0.02 two tailed (family-wise uncorrected). TH: thalamus; STR: striatum; LCN: lateral cortical network; HCP: hippocampus; DMN: default mode network. 
A
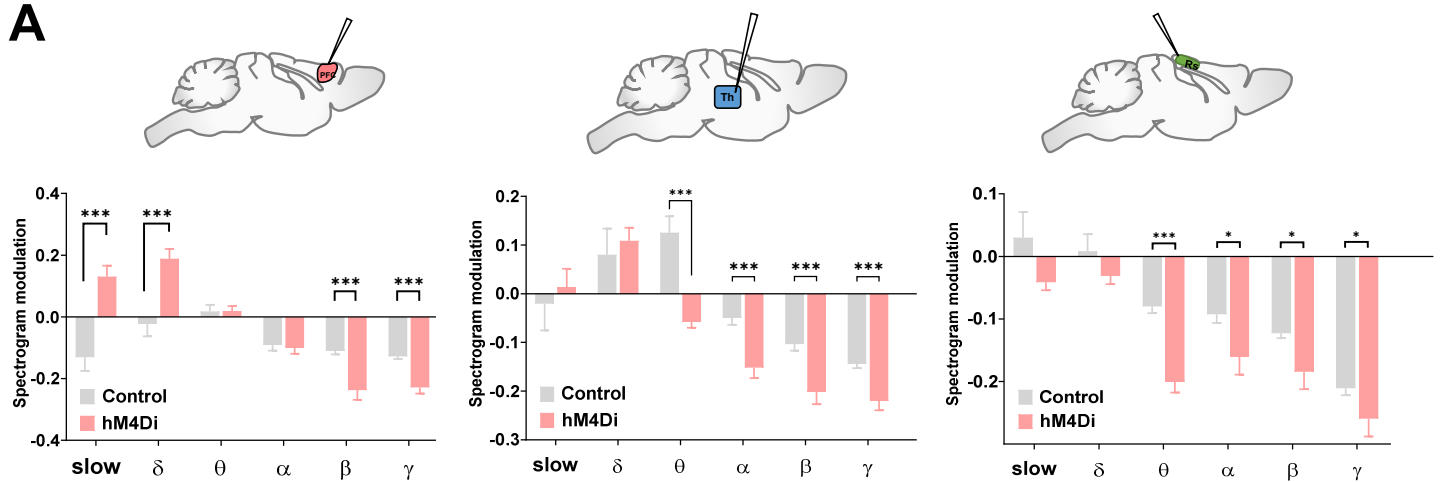

B
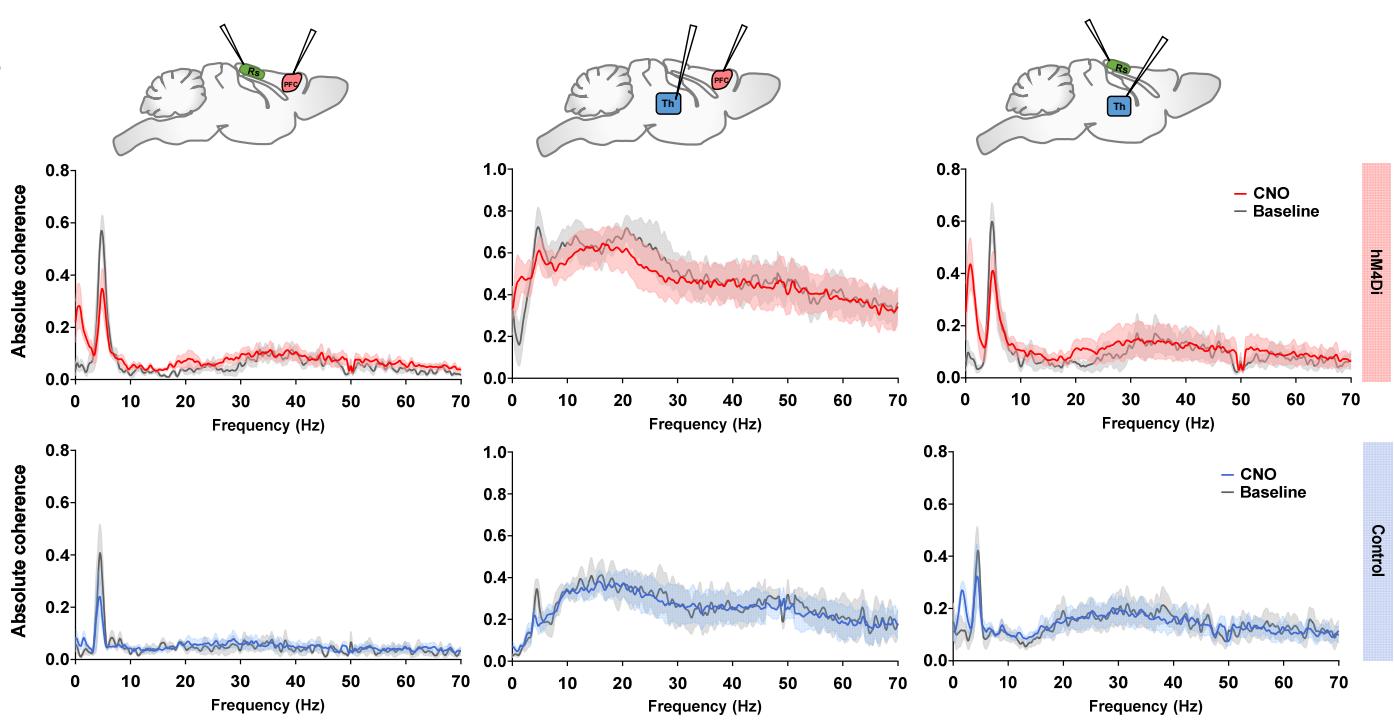

Figure S6. Power spectra and raw absolute coherence in multi-electrode recordings

(A) Quantification of band-specific power spectrum changes in LFPs recorded in the PFC (left), centromedial thalamus (Th; center) and retrosplenial cortex (Rs; right) upon systemic CNO administration. Power was quantified with respect to pre-injection baseline (mean \pm SEM; ${ }^{*} p<0.05$, $* * p<0.01, * * * p<0.001$, t-test, FDR corrected).

(B) Mean absolute power coherence for all pairs of electrophysiologically-probed regions (PFC-Rs; PFC-Th; Rs-Th) during both baseline and CNO active time window in hM4Di and control animals (mean \pm SEM). 

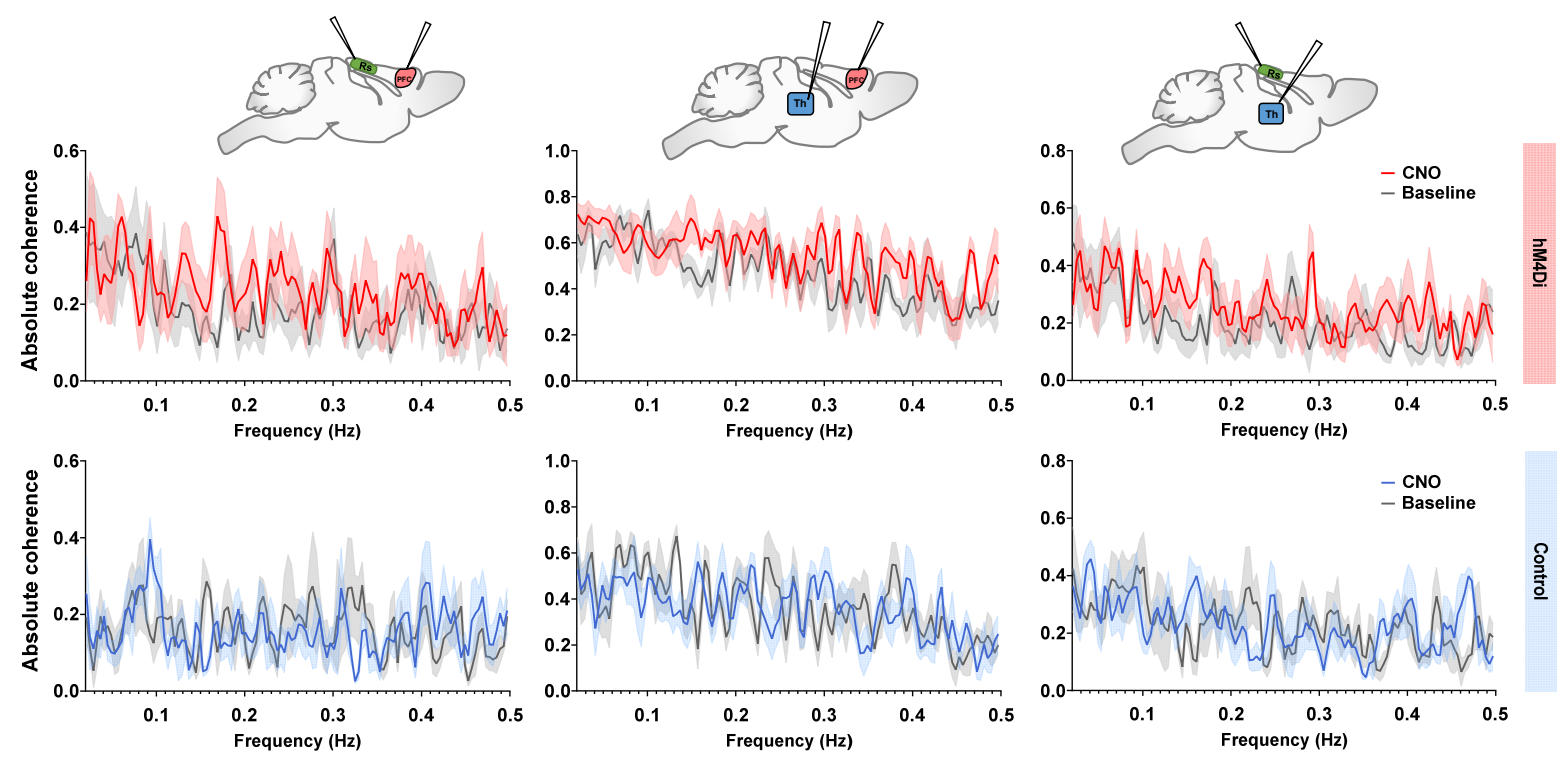

Figure S7. Mean ultra-slow oscillatory coherence in control and hM4Di subjects

Mean absolute power coherence of ultra-slow oscillation for all pairs of electrophysiologically-probed regions (PFC-Rs; PFC-Th; Rs-Th) computed for both baseline and CNO active time window in hM4Di and control animals (mean \pm SEM). 

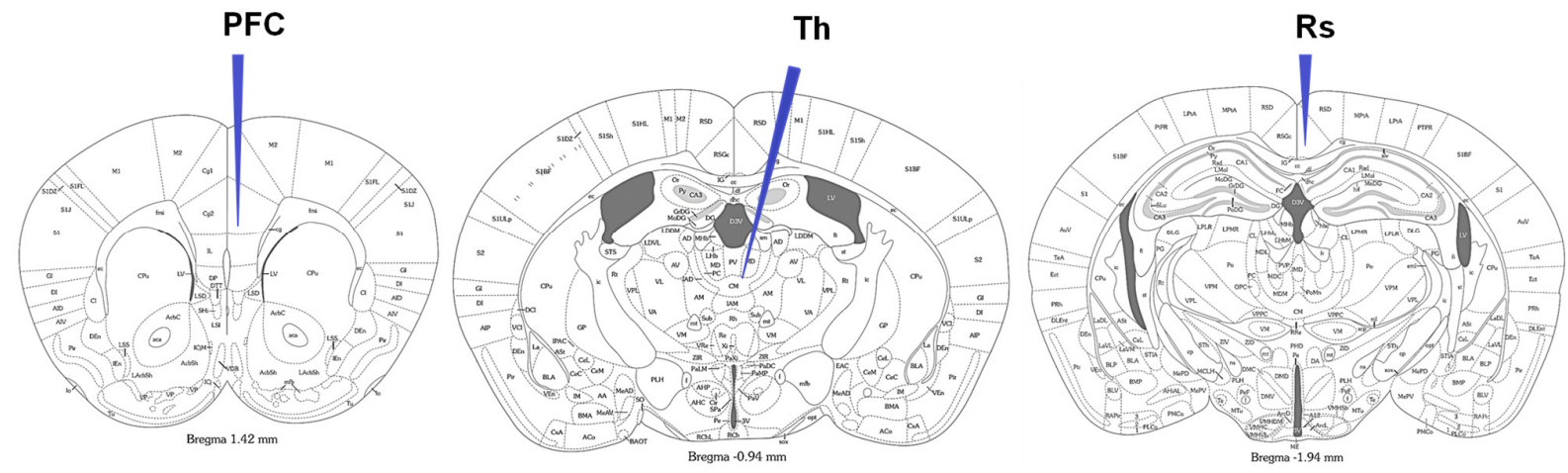

Figure S8. Anatomical location of electrode insertion site for multi-electrode neural recordings. 


\section{References}

1. Power, J., Schlaggar, B. \& Petersen, S. Studying Brain Organization via Spontaneous fMRI Signal. Neuron 84, 681-696 (2014).

2. Hagmann, P., et al. Mapping the structural core of human cerebral cortex. PLoS Biol 6 (2008).

3. Wang, Z., et al. The Relationship of Anatomical and Functional Connectivity to Resting-State Connectivity in Primate Somatosensory Cortex. Neuron 78, 1116-1126 (2013).

4. Coletta, L., et al. Network structure of the mouse brain connectome with voxel resolution. Science Advances, 2020.2003.2006.973164 (2020).

5. Honey, C.J., et al. Predicting human resting-state functional connectivity from structural connectivity. Proceedings of the National Academy of Sciences 106, 2035-2040 (2009).

6. Goni, J., et al. Resting-brain functional connectivity predicted by analytic measures of network communication. Proceedings of the National Academy of Sciences of the United States of America 111, 833-838 (2014).

7. O'Reilly, J.X., et al. Causal effect of disconnection lesions on interhemispheric functional connectivity in rhesus monkeys. Proceedings of the National Academy of Sciences 110, 1398213987 (2013).

8. Grayson, D.S., et al. The Rhesus Monkey Connectome Predicts Disrupted Functional Networks Resulting from Pharmacogenetic Inactivation of the Amygdala. Neuron 91, 453-466 (2016).

9. Ponce-Alvarez, A., et al. Resting-State Temporal Synchronization Networks Emerge from Connectivity Topology and Heterogeneity. PLoS Comput Biol 11, e1004100 (2015).

10. Alstott, J., Breakspear, M., Hagmann, P., Cammoun, L. \& Sporns, O. Modeling the Impact of Lesions in the Human Brain. PLOS Computational Biology 5, e1000408 (2009). 
11. Gent, T.C., Bandarabadi, M., Herrera, C.G. \& Adamantidis, A.R. Thalamic dual control of sleep and wakefulness. Nature Neuroscience 21, 974-984 (2018).

12. Liu, X., et al. Subcortical evidence for a contribution of arousal to fMRI studies of brain activity. Nature Communications 9, 395 (2018).

13. Gutierrez-Barragan, D., Basson, M.A., Panzeri, S. \& Gozzi, A. Infraslow State Fluctuations Govern Spontaneous fMRI Network Dynamics. Current Biology 29, 2295-2306.e2295 (2019).

14. Yousefi, B., Shin, J., Schumacher, E.H. \& Keilholz, S.D. Quasi-periodic patterns of intrinsic brain activity in individuals and their relationship to global signal. Neurolmage 167, 297-308 (2018).

15. Sforazzini, F., et al. Altered functional connectivity networks in acallosal and socially impaired BTBR mice. Brain Struct Funct 221, 941-954 (2016).

16. Tyszka, J.M., Kennedy, D.P., Adolphs, R. \& Paul, L.K. Intact Bilateral Resting-State Networks in the Absence of the Corpus Callosum. The Journal of Neuroscience 31, 15154-15162 (2011).

17. Eldaief, M.C., Halko, M.A., Buckner, R.L. \& Pascual-Leone, A. Transcranial magnetic stimulation modulates the brain's intrinsic activity in a frequency-dependent manner. Proceedings of the National Academy of Sciences 108, 21229 (2011).

18. Pagani, M., et al. A cross-species link between mTOR-related synaptic pathology and functional hyperconnectivity in autism. bioRxiv, 2020.2010.2007.329292 (2020).

19. Hillary, F.G. \& Grafman, J.H. Injured brains and adaptive networks: the benefits and costs of hyperconnectivity. Trends in cognitive sciences 21, 385-401 (2017).

20. Siegel, J.S., et al. Disruptions of network connectivity predict impairment in multiple behavioral domains after stroke. Proceedings of the National Academy of Sciences 113, E4367E4376 (2016).

21. Giorgi, A., et al. Brainwide mapping of endogenous serotonergic transmission via chemogenetic-fMRI. Cell Reports (2017). 
22. Whitesell, J.D., et al. Regional, layer, and cell-class specific connectivity of the mouse default mode network. Neuron, 2020.2005.2013.094458 (2020).

23. Suárez, L.E., Markello, R.D., Betzel, R.F. \& Misic, B. Linking Structure and Function in Macroscale Brain Networks. Trends in Cognitive Sciences (2020).

24. Xue, M., Atallah, B.V. \& Scanziani, M. Equalizing excitation-inhibition ratios across visual cortical neurons. Nature 511, 596 (2014).

25. Beier, K.T., et al. Rabies screen reveals GPe control of cocaine-triggered plasticity. Nature 549, 345 (2017).

26. Bertero, A., et al. Autism-associated 16p11.2 microdeletion impairs prefrontal functional connectivity in mouse and human BRAIN 141, 2055-2065 (2018).

27. Pagani, M., et al. Deletion of autism risk gene Shank3 disrupts prefrontal connectivity. The Journal of Neuroscience, 2529-2518 (2019).

28. Wiegert, J.S., Mahn, M., Prigge, M., Printz, Y. \& Yizhar, O. Silencing Neurons: Tools, Applications, and Experimental Constraints. Neuron 95, 504-529 (2017).

29. Jendryka, M., et al. Pharmacokinetic and pharmacodynamic actions of clozapine-N-oxide, clozapine, and compound 21 in DREADD-based chemogenetics in mice. Scientific Reports 9, 4522 (2019).

30. Trakoshis, S., et al. Intrinsic excitation-inhibition imbalance affects medial prefrontal cortex differently in autistic men versus women. eLife 9, e55684 (2020).

31. Turchi, J., et al. The Basal Forebrain Regulates Global Resting-State fMRI Fluctuations. Neuron 97, 940-952.e944 (2018).

32. Mitchell, A.S. The mediodorsal thalamus as a higher order thalamic relay nucleus important for learning and decision-making. Neuroscience \& Biobehavioral Reviews 54, 76-88 (2015). 
33. Schleifer, C.H., et al. Reciprocal Disruptions in Thalamic and Hippocampal Resting-State Functional Connectivity in Youth with 22q11.2 Deletions. bioRxiv (2017).

34. Hughes, S.W., Lőrincz, M.L., Parri, H.R. \& Crunelli, V. Chapter 10 - Infraslow $(<0.1 \mathrm{~Hz})$ oscillations in thalamic relay nuclei: basic mechanisms and significance to health and disease states. in Progress in Brain Research (ed. E.J.W. Van Someren, Y.D. Van Der Werf, P.R. Roelfsema, H.D. Mansvelder \& F.H. Lopes Da Silva) 145-162 (Elsevier, 2011).

35. He, B.J., Snyder, A.Z., Zempel, J.M., Smyth, M.D. \& Raichle, M.E. Electrophysiological correlates of the brain's intrinsic large-scale functional architecture. Proceedings of the National Academy of Sciences of the United States of America 105, 16039-16044 (2008).

36. Nir, Y., et al. Interhemispheric correlations of slow spontaneous neuronal fluctuations revealed in human sensory cortex. Nature Neuroscience 11, 1100-1108 (2008).

37. van Kerkoerle, T., et al. Alpha and gamma oscillations characterize feedback and feedforward processing in monkey visual cortex. Proceedings of the National Academy of Sciences $111,14332-14341$ (2014).

38. Besserve, M., Sch+! lkopf, B., Logothetis, N.K. \& Panzeri, S. Causal relationships between frequency bands of extracellular signals in visual cortex revealed by an information theoretic analysis. Journal of computational neuroscience 29, 547-566 (2010).

39. Bosman, Conrado A., et al. Attentional Stimulus Selection through Selective Synchronization between Monkey Visual Areas. Neuron 75, 875-888 (2012).

40. Mateo, C., Knutsen, P.M., Tsai, P.S., Shih, A.Y. \& Kleinfeld, D. Entrainment of Arteriole Vasomotor Fluctuations by Neural Activity Is a Basis of Blood-Oxygenation-Level-Dependent “Resting-State" Connectivity. Neuron 96, 936-948.e933 (2017). 
41. Wang, L., Saalmann, Yuri B., Pinsk, Mark A., Arcaro, Michael J. \& Kastner, S.

Electrophysiological Low-Frequency Coherence and Cross-Frequency Coupling Contribute to BOLD Connectivity. Neuron 76, 1010-1020 (2012).

42. Bowyer, S.M. Coherence a measure of the brain networks: past and present. Neuropsychiatric Electrophysiology 2, 1 (2016).

43. Friston, K.J. \& Büchel, C. Functional connectivity: eigenimages and multivariate analyses. Statistical parametric mapping: the analysis of functional brain images, 492-507 (2006).

44. Pan, W.-J., Thompson, G.J., Magnuson, M.E., Jaeger, D. \& Keilholz, S. Infraslow LFP correlates to resting-state fMRI BOLD signals. Neurolmage 74, 288-297 (2013).

45. Busche, M.A. \& Konnerth, A. Impairments of neural circuit function in Alzheimer's disease. Philosophical Transactions of the Royal Society B: Biological Sciences 371, 20150429 (2016).

46. Pusil, S., et al. Hypersynchronization in mild cognitive impairment: the ' $X$ ' model. Brain 142, 3936-3950 (2019).

47. Safaai, H., Neves, R., Eschenko, O., Logothetis, N.K. \& Panzeri, S. Modeling the effect of locus coeruleus firing on cortical state dynamics and single-trial sensory processing. Proceedings of the National Academy of Sciences 112, 12834-12839 (2015).

48. Reimann, H.M. \& Niendorf, T. The (Un)Conscious Mouse as a Model for Human Brain Functions: Key Principles of Anesthesia and Their Impact on Translational Neuroimaging. Frontiers in Systems Neuroscience 14 (2020).

49. Dubovik, S., et al. The behavioral significance of coherent resting-state oscillations after stroke. Neurolmage 61, 249-257 (2012).

50. Ranasinghe, K.G., et al. Neurophysiological signatures in Alzheimer's disease are distinctly associated with TAU, amyloid- $\beta$ accumulation, and cognitive decline. Science Translational Medicine 12, eaaz4069 (2020). 
51. Huth, A.G., Nishimoto, S., Vu, A.T. \& Gallant, J.L. A continuous semantic space describes the representation of thousands of object and action categories across the human brain. Neuron 76, 1210-1224 (2012).

52. Hughes, S.W., Cope, D.W., Blethyn, K.L. \& Crunelli, V. Cellular Mechanisms of the Slow (<1 Hz) Oscillation in Thalamocortical Neurons In Vitro. Neuron 33, 947-958 (2002).

53. David, F., et al. Essential Thalamic Contribution to Slow Waves of Natural Sleep. The Journal of Neuroscience 33, 19599-19610 (2013).

54. Narikiyo, K., et al. The claustrum coordinates cortical slow-wave activity. Nature Neuroscience (2020).

55. Lu, H., et al. Synchronized delta oscillations correlate with the resting-state functional MRI signal. Proc.Natl.Acad.Sci.U.S.A 104, 18265-18269 (2007).

56. Lu, H., et al. Low- but Not High-Frequency LFP Correlates with Spontaneous BOLD Fluctuations in Rat Whisker Barrel Cortex. Cerebral Cortex (2014).

57. Neuner, I., et al. The Default Mode Network and EEG Regional Spectral Power: A Simultaneous fMRI-EEG Study. PLOS ONE 9, e88214 (2014).

58. Chan, R.W., et al. Low-frequency hippocampal-cortical activity drives brain-wide restingstate functional MRI connectivity. Proceedings of the National Academy of Sciences 114, E6972E6981 (2017).

59. Franks, N.P. General anaesthesia: from molecular targets to neuronal pathways of sleep and arousal. Nature Reviews Neuroscience 9, 370 (2008).

60. Watanabe, T., et al. Bidirectional effects on interhemispheric resting-state functional connectivity induced by excitatory and inhibitory repetitive transcranial magnetic stimulation. Human Brain Mapping 35, 1896-1905 (2014). 
61. Strens, L.H.A., et al. The effects of subthreshold $1 \mathrm{~Hz}$ repetitive TMS on cortico-cortical and interhemispheric coherence. Clinical Neurophysiology 113, 1279-1285 (2002).

62. Peeters, L.M., et al. Chemogenetic silencing of neurons in the mouse anterior cingulate area modulates neuronal activity and functional connectivity. Neurolmage 220, 117088 (2020).

63. Tu, W., Ma, Z., Ma, Y., Dopfel, D. \& Zhang, N. Suppressing Anterior Cingulate Cortex Modulates Default Mode Network and Behavior in Awake Rats. Cerebral Cortex (2020).

64. Markicevic, M., et al. Cortical excitation:inhibition imbalance causes network-specific functional hypoconnectivity: a DREADD-fMRI study. Cerebral cortex, 492108 (2018).

65. Carlén, M. What constitutes the prefrontal cortex? Science 358, 478-482 (2017).

66. Vogt, B. \& Paxinos, G. Cytoarchitecture of mouse and rat cingulate cortex with human homologies. Brain Struct Funct 219, 185-192 (2014).

67. Paxinos, G. \& Franklin, K. The Mouse Brain in Stereotaxic Coordinates (Academic Press, Sydney, 2003).

68. Sforazzini, F., Schwarz, A.J., Galbusera, A., Bifone, A. \& Gozzi, A. Distributed BOLD and CBVweighted resting-state networks in the mouse brain. Neuroimage 87, 403-415 (2014).

69. Ferrari, L., et al. A robust experimental protocol for pharmacological fMRI in rats and mice. J Neurosci Methods 204, 9-18 (2012).

70. Gozzi, A., et al. A multimodality investigation of cerebral hemodynamics and autoregulation in pharmacological MRI. Magn Reson Imaging 25, 826-833 (2007).

71. Liska, A., Galbusera, A., Schwarz, A.J. \& Gozzi, A. Functional connectivity hubs of the mouse brain. Neuroimage 115, 281-291 (2015).

72. Wu, G.R., et al. A blind deconvolution approach to recover effective connectivity brain networks from resting state fMRI data. Medical Image Analysis 17, 365-374 (2013). 
73. Belitski, A., et al. Low-frequency local field potentials and spikes in primary visual cortex convey independent visual information. J Neurosci 28, 5696-5709 (2008).

74. Welch, P. The use of fast Fourier transform for the estimation of power spectra: A method based on time averaging over short, modified periodograms. IEEE Transactions on Audio and Electroacoustics 15, 70-73 (1967). 


\section{Figures}

A

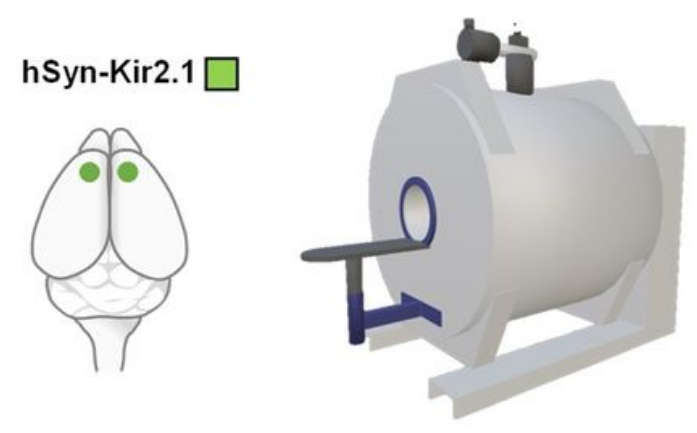

C

Difference - Kir2.1 > Control
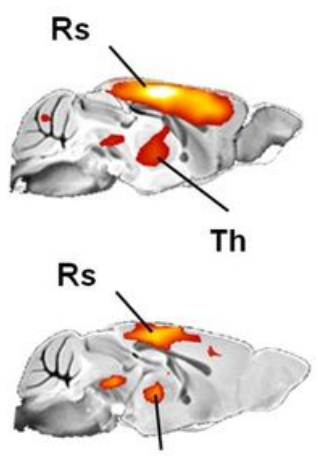

Th

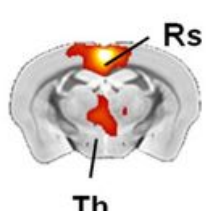

Th

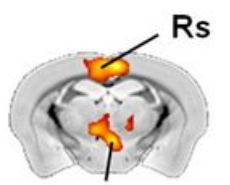

Th
B

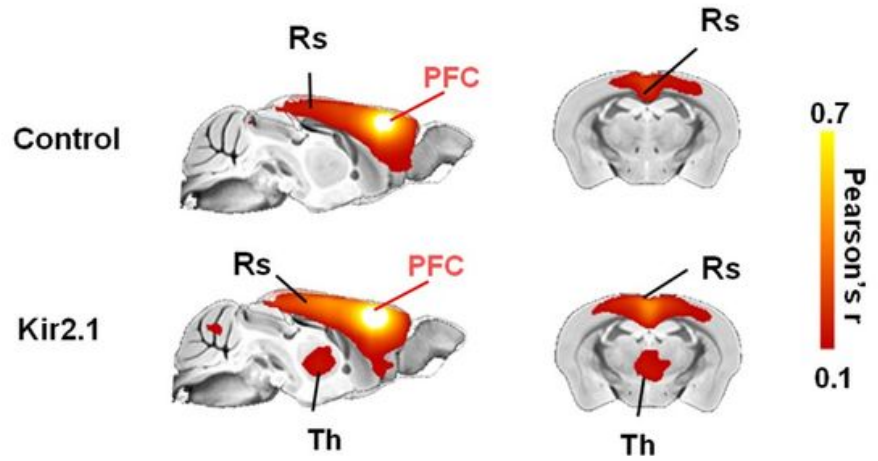

D

E
0.7
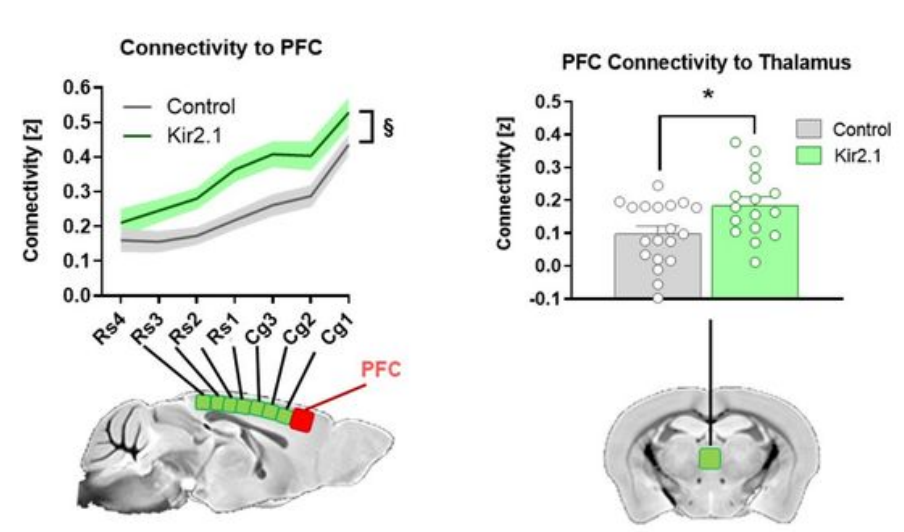

F

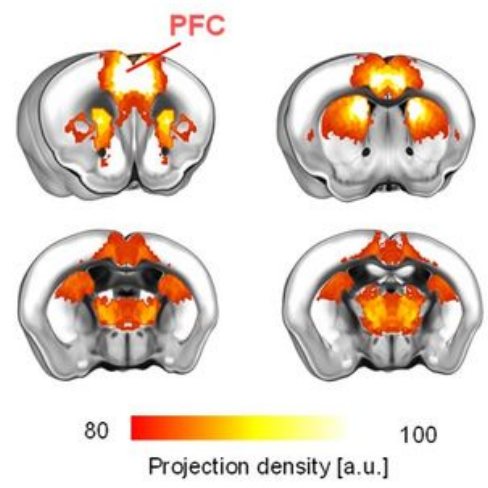

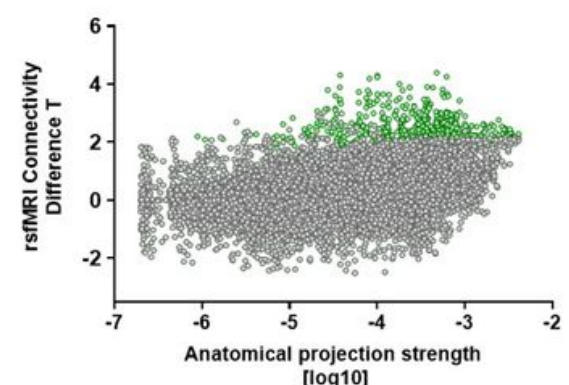

[log10]

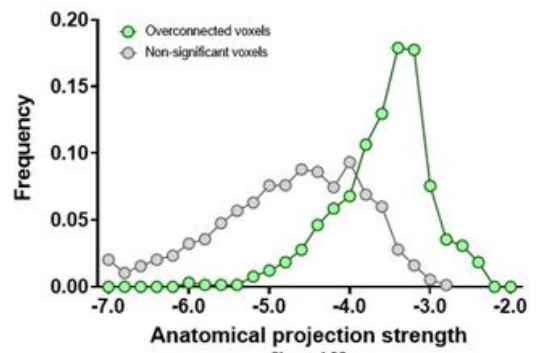

[ $\log 10]$

\section{Figure 1}

Chronic silencing of the mouse PFC results in rsfMRI overconnectivity (A) Summary of experimental design. The potassium channel Kir2.1 $(n=16)$ or GFP (control, $n=19)$ were transduced bilaterally into the PFC of adult male mice. Four weeks after viral injections, mice underwent rsfMRI scanning. (B) Seed based connectivity mapping of the PFC in GFP (control), and Kir2.1》transduced subjects. (C) Corresponding group difference maps. Area with significantly increased rsfMRI connectivity in Kir2.1 expressing mice are depicted in red『yellow ( $r$ and T stat difference map). (D) Antero囚posterior

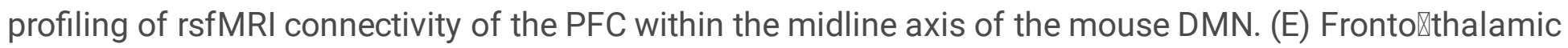
rsfMRI overconnectivity in Kir2.1 expressing mice. (F) Regions exhibiting rsfMRI overconnectivity in 
Kir2.1 mice are robustly innervated by the PFC. Left: Axonal projections from the PFC (top $20 \%$ strongest connections). Middle: scatter plot illustrating intergroup differences in rsfMRI connectivity as a function of PFC structural connectivity strength. Green dots indicate significantly functionally overconnected voxels. Right: Distribution of overconnected voxels as a function of axonal connectivity strength Green. DMN: Default Mode Network; Cg: cingulate cortex; PFC: prefrontal cortex, RS: retrosplenial; Th: Thalamus. $\S p<0.05,2 \rrbracket$ way ANOVA, repeated measurements; * $p<0.05$, student $t$ test.

A

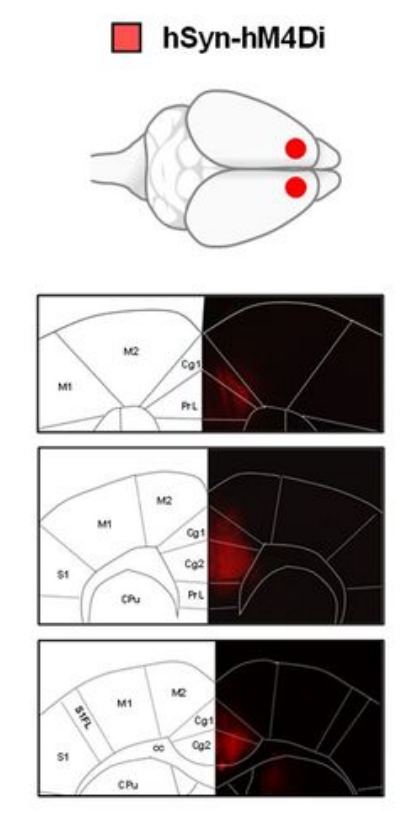

Viral transfection
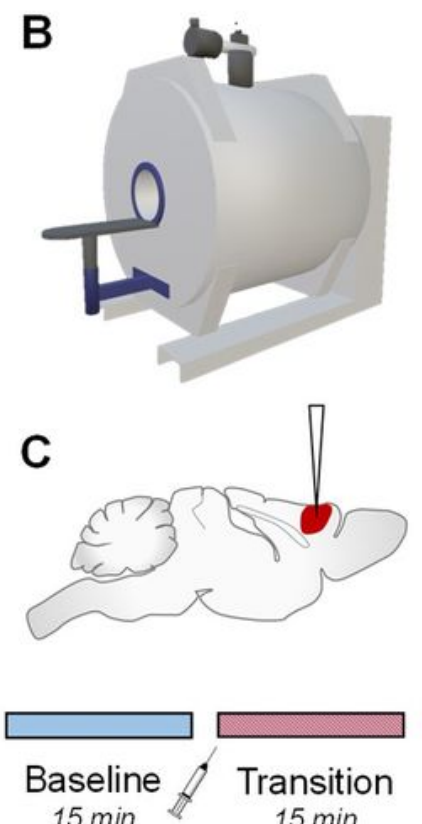

$15 \mathrm{~min}$
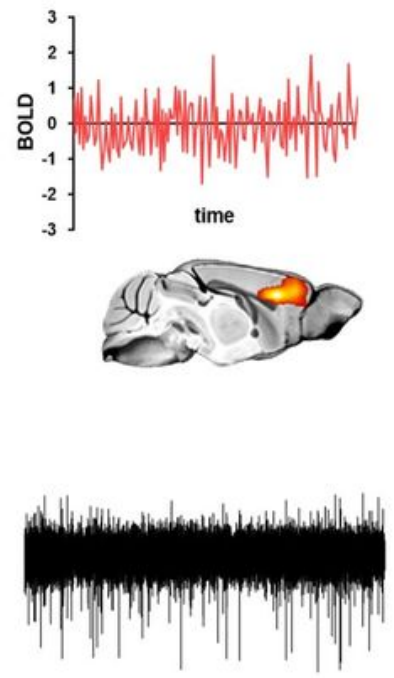

Active

$35 \mathrm{~min}$

\section{rsfMRI and electrophysiological recordings}

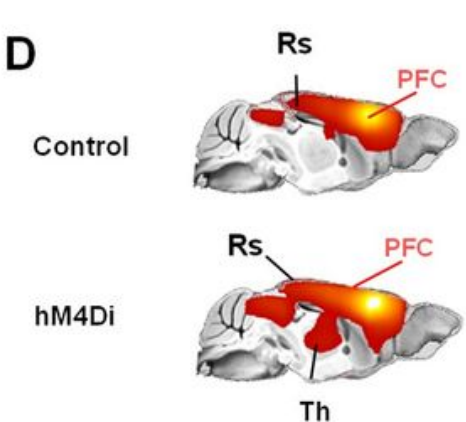

E

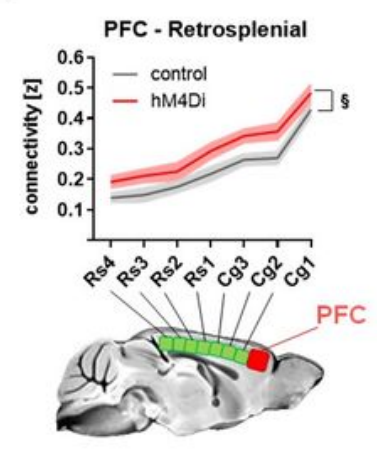

$\mathbf{F}$

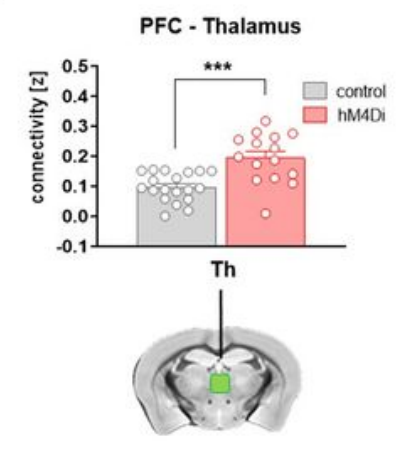

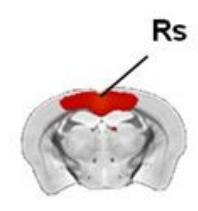

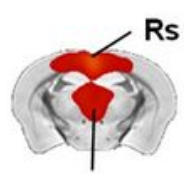

Th

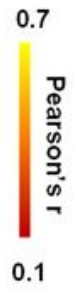

0.1

G

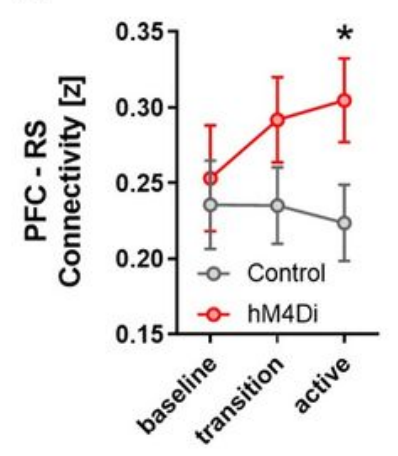

Figure 2 
Chemogenetic silencing of the mouse PFC results in rsfMRI overconnectivity. (A) Experimental design of

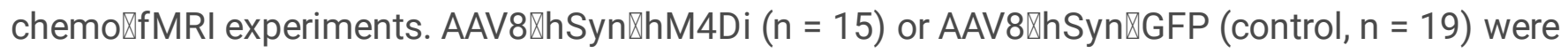
bilaterally injected into the PFC of wild type. The bottom panels show representative viral expression across three adjacent PFC slices three weeks after transduction (B) mice underwent rsfMRI scanning or (C) electrophysiological recordings. A reference acquisition timeline is reported to depict timeseries binning into a $15 \mathrm{~min}$ preखCNO reference baseline, a drug equilibration window (15 min, transition), and a 35 min CNO active time window (active). (D) Seed $\triangle$ based connectivity of the PFC and between group difference map revealed rsfMRI over囚connectivity in the DMN of hM4Di expressing mice during the active phase. (E) Antero囚posterior profiling of rsfMRI connectivity of the PFC along the midline axis of the mouse DMN in the two cohorts. (F) Thalamo®cortical rsfMRI hyper synchronization in hM4Di expressing mice. (G) Prefrontal『retrosplenial and prefrontal】thalamic connectivity timecourse. Data are plotted as mean \pm SEM. Cg: cingulate cortex; PFC: prefrontal cortex, RS: retrosplenial cortex; Th: Thalamus. $\S p<0.05$, 2\way ANOVA repeated measurements, genotype effect; * $p<0.05$, ***
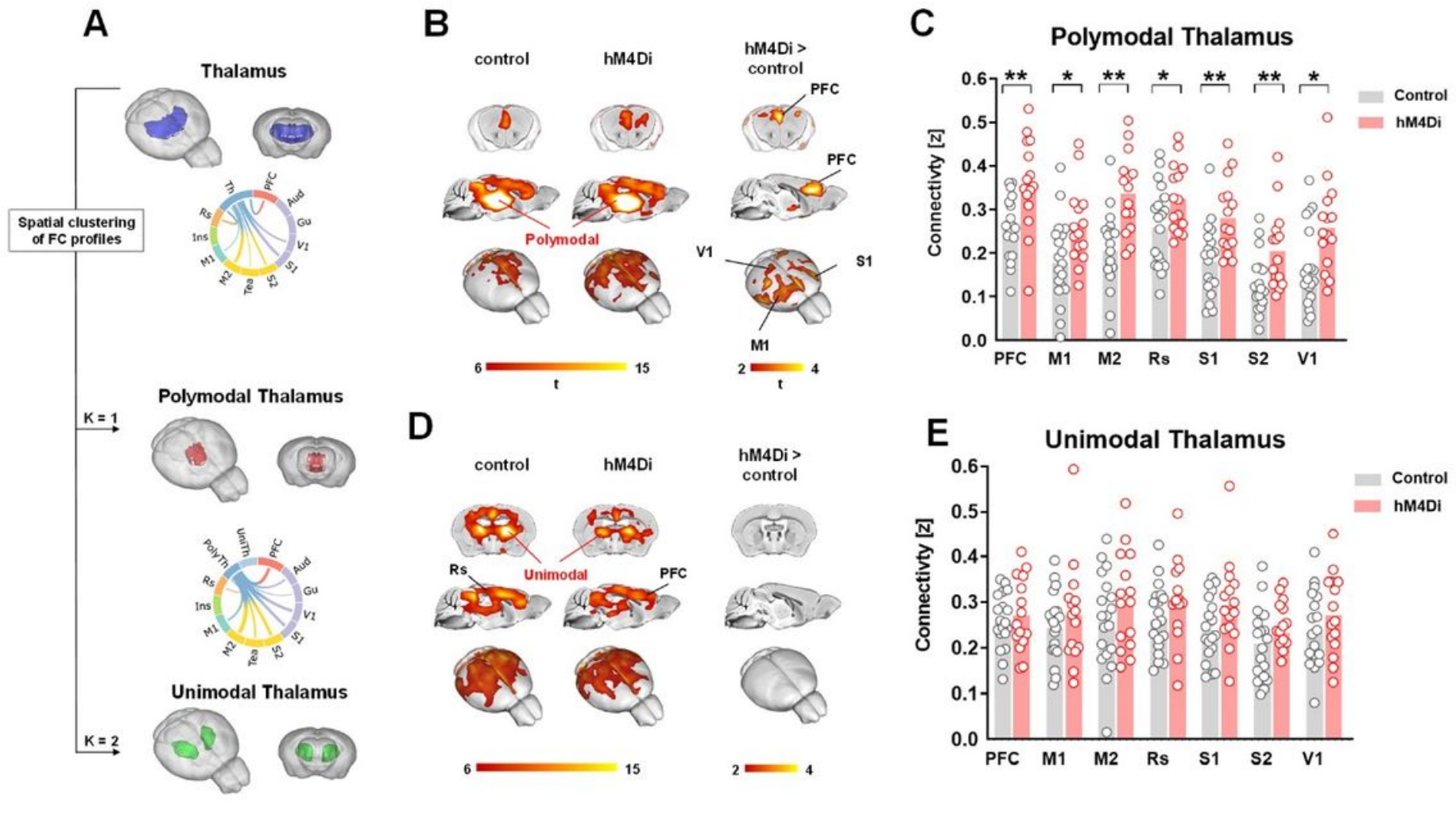

\section{Figure 3}

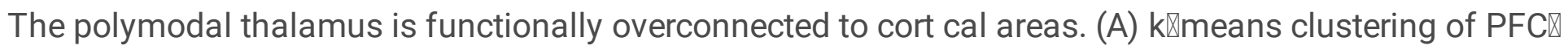
thalamic rsfMRI connectivity profiles (thalamus, blue; unimodal thalamus, green; polymodal thalamus, red) and circular representation of between $₫$ group connectivity difference of the entire thalamus and thalamic partition (UniTh: unimodal; PolyTh: polymodal thalamus) in Control $(n=19)$ and hM4Di $(n=15)$ animals. (B囚D) Seed connectivity of sub囚thalamic partitions, between group difference maps (B囚C polymodal thalamus; $\mathrm{D} \otimes \mathrm{E}$, unimodal thalamus) and corresponding quantification of thalamo®cortical connectivity $\left({ }^{\star} \mathrm{p}<0.05,{ }^{* \star} \mathrm{p}<0.01\right.$; Benjamini冈Hochberg corrected). 
A

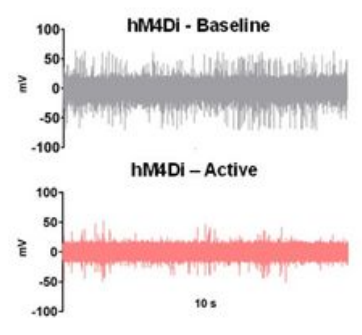

D
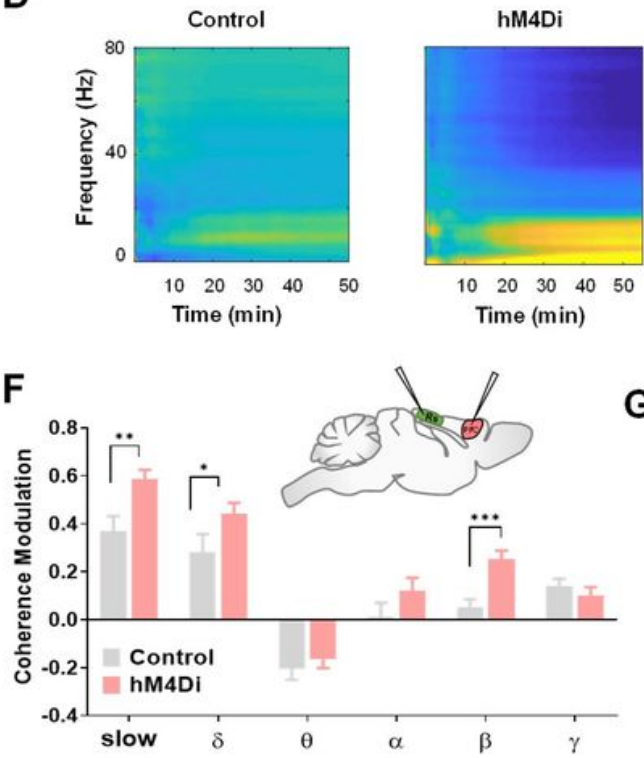

I

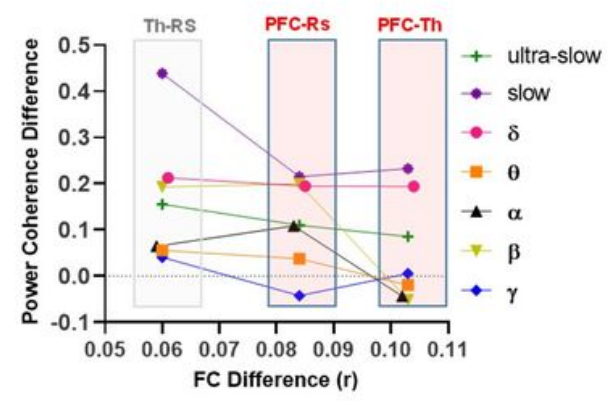

B
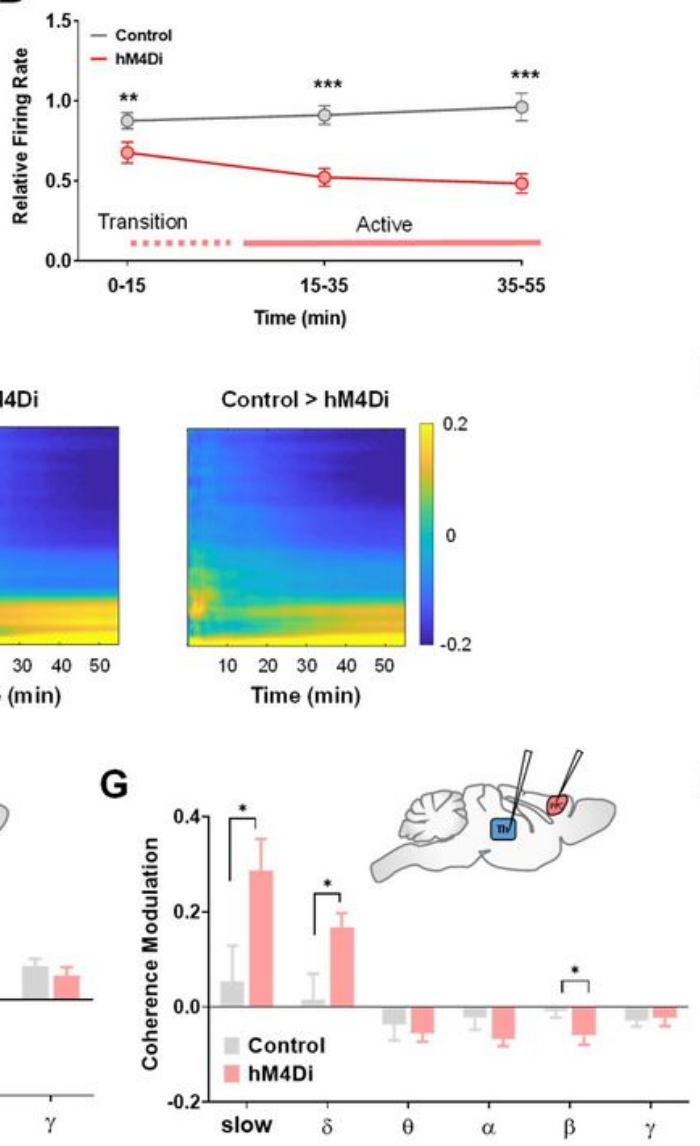

L

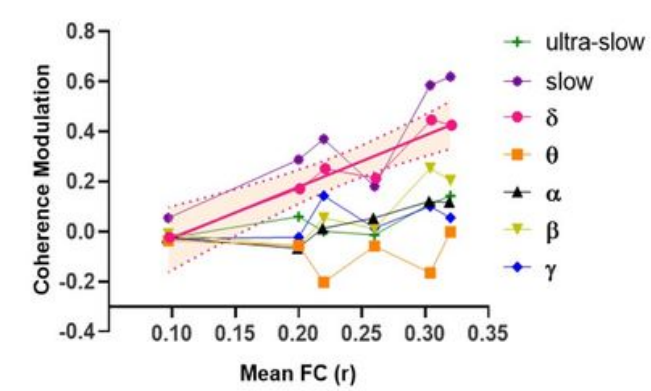

C
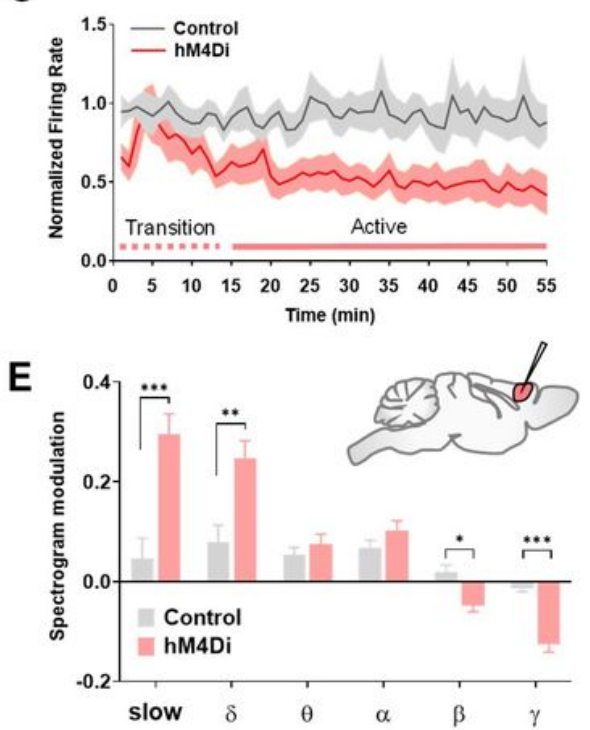

$\mathbf{H}$

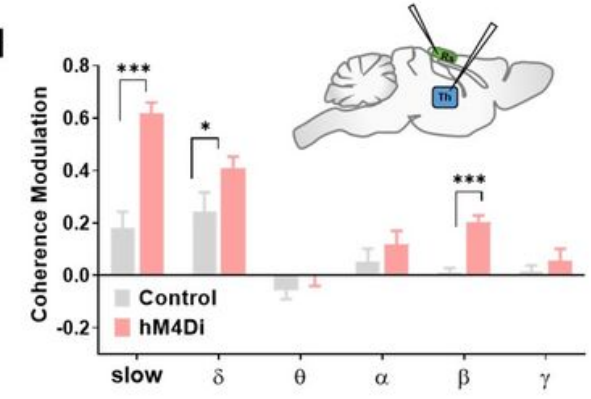

M

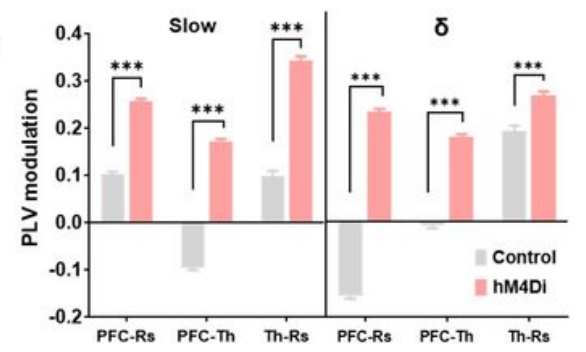

\section{Figure 4}

Chemogenetic silencing of the PFC results in decreased $\nabla$ activity and increased interareal slow oscillatory coherence. (A) Representative raw traces collected before and after CNO injection in a representative recordings site of hM4Di animal. (B囚C) Reduced firing rate in hM4Di囚expressing mice (n $=5$ ) compared to GFP $₫$ transduced controls $(n=5)$. (D) Mean post $₫$ injection spectrogram in control (Left), hM4Di囚expressing animals (center), and mean between group difference (right). (E) Quantification of band \specific power spectrum changes upon CNO injection in both groups $(\mathrm{F} \otimes G \otimes H)$. Baseline normalized power coherence at different frequency bands for PFC区Retrosplenial (Left), PFCthalamus and

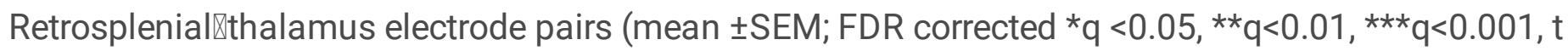
test). (I) Band specific coherence and mean functional connectivity (FC) difference (hM4Di - Control) for all pairs of electrophysiologically邓probed regions. Mean FC data were extracted for corresponding 
regional pairs (Fig. 2) during the $\mathrm{CNO}$ active time window in $\mathrm{hM} 4 \mathrm{Di}$ and control animals. (L) Correlation between corresponding band specific coherence and mean functional connectivity for all pairs of

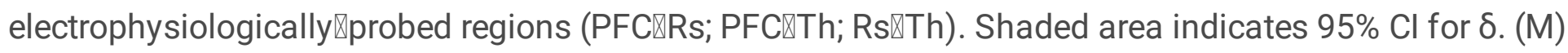
Baseline normalized phase coherence in slow and delta band between electrode pairs (mean $\pm S E M ; * p$ $<0.05,{ }^{\star *} p<0.01,{ }^{* \star *} \mathrm{p}<0.001, \mathrm{t}$ test, FDR corrected). 